\title{
Der Sahara-Konflikt und die Krise der Organisation der Afrika- nischen Einheit (OAU)
}

von Jürgen Taeger

\section{Vorbemerkung}

Die Organisation der Afrikanischen Einheit (OAU) steckt derzeit in der tiefsten Krise seit ihrer Gründung im Jahr 1963. Erstmals konnte 1982 die nach Tripolis einberufene Versammlung ihrer Staats- und Regierungschefs trotz intensiver diplomatischer Anstrengungen nicht abgehalten werden. Sowohl im August als auch im November 1982 scheiterten die Sitzungseröffnungen an der Abwesenheit zahlreicher Delegationen; das nach der Satzung erforderliche 2/3-Quorum konnte nicht erreicht werden. Die verspätete Durchführung der 19. Gipfelkonferenz vom 8.-12. Juni 1983 in Addis Abeba nun mit der 'Rekord-Beteiligung von 49 Mitgliedsstaaten - kann nicht über die schwierige Lage hinwegtäuschen, in der sich diese bedeutende, der Welt mitgliederstärkste Regionalorganisation befindet. Kenias Präsident Daniel Arap Moi, bis zum Gipfeltreffen 1983 Vorsitzender der OAU, bezeichnete in seiner Eröffnungsansprache allein schon die Tatsache der Zusammenkunft nach zwei vergeblichen Anläufen als einen Sieg für Afrika. ${ }^{1}$ Er brachte damit in eindringlicher Weise zum Ausdruck, daß die reale Gefahr eines Zusammenbruchs erst im letzten Moment abgewendet werden konnte. In Anbetracht der vermutlich destruktiven Wirkung eines Kollapses der Organisation auf den afrikanischen Emanzipationsprozeß treten die Aktivitäten der OAU und ihrer 16 Unterorganisationen auf politischem, ökonomischem, humanitärem und sozialem Gebiet als Gegenstand öf fentlicher Aufmerksamkeit zurück. Nach der Feststellung eines Beobachters "liegt die Kraft der OAU vor allem in der Tatsache, daß sie, trotz der unzähligen Fehlschläge und Meinungsverschiedenheiten, auch weiterhin besteht ". ${ }^{2}$

Die Auseinandersetzungen in der OAU haben sich besonders an einem Objekt, mit dem sich die OAU bereits seit ihrer Gründung kontinuierlich beschäftigt, entzündet,nämlich dem Konflikt um die ehemalige spanische Kolonie Sahara. Die Aufnahme der 'Demokratischen Arabischen Republik Sahara in die afrikanische Staatengemeinschaft durch

Le monde, 10. 6. 1983.

Matthews, Krise in der OAE, in: Internationale Politik, (1983)686, S. 13-21(14); zur OAU als "Motor des afrikanischen Integrationsprozesses, Forum innerafrikanischer, Streitigkeiten und Katalysator zur Gewinnung kontinentaler Positionen im Verhältnis zu nicht-afrikanischen Staaten" ausführlich Kunig, Die Organisation der afrikanischen Einheit und der Prozeß des Nation Building: Die völkerrechtlichen Rahmenbedingungen, in: Archiv des Völkerrechts, (1982)2, S. 40-57. 
Demokratische Arabische Republik Sahara

N

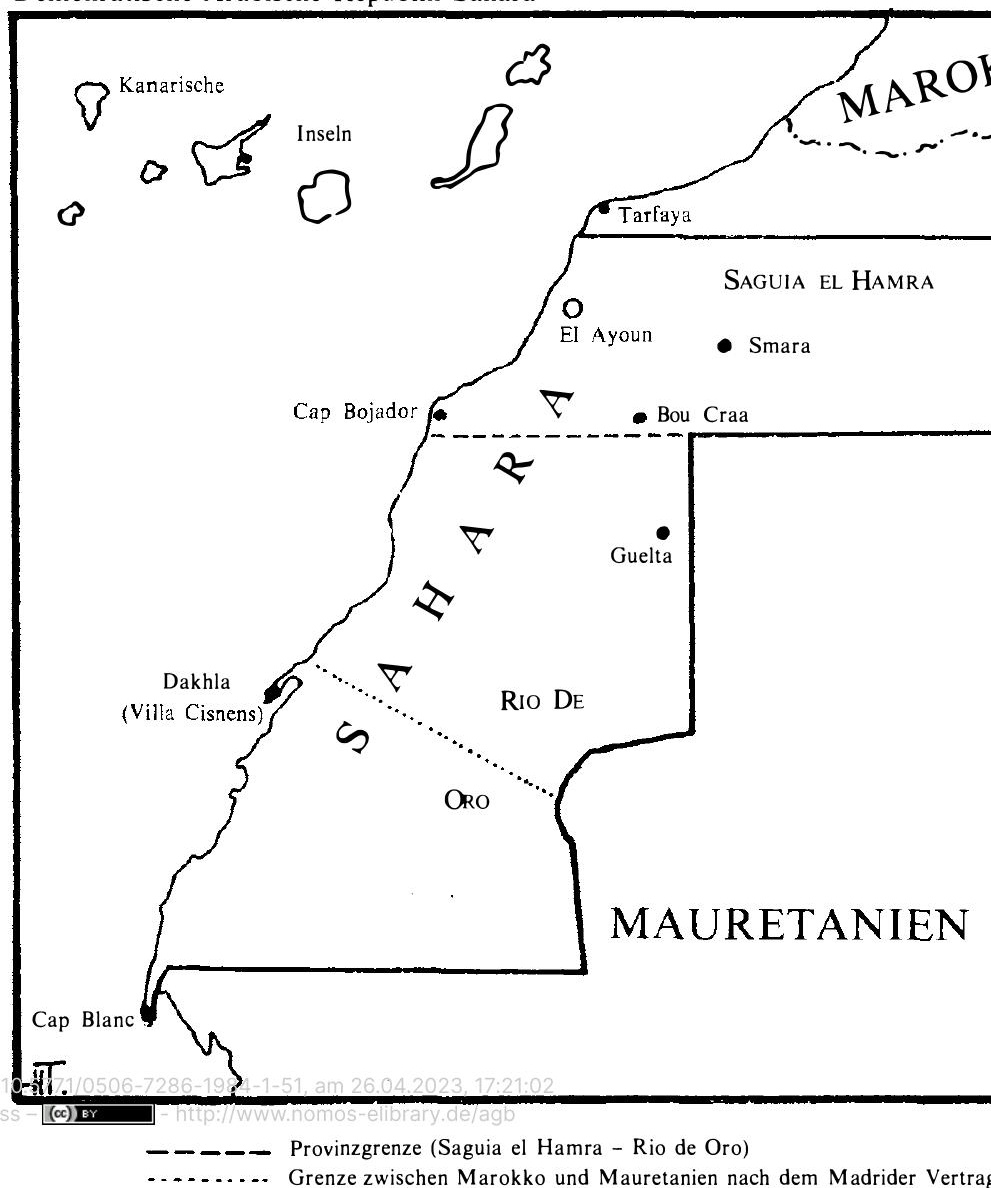


die absolute Mehrheit ihrer Mitglieder stellt die OAU vor die größte Zerreißprobe ihrer Geschichte. Der folgende Beitrag skizziert die historische Entwicklung und den aktuellen Stand dieses Konflikts und untersucht die Bemühungen um seine Beilegung.

\section{Zur Geschichte der Westsahara}

Spanische Expeditionen betraten den af rikanischen Kontinent in Höhe der Westsahara bereits im 15. Jahrhundert. Insbesondere von den Kanarischen Inseln aus betrieben Spanier in den folgenden Jahrhunderten den Handel mit afrikanischen Küstenbewohnern. Von einer spanischen Kolonialzeit kann aber frühestens ab 1884 gesprochen werden. ${ }^{3} \mathrm{Zu}$ dieser Zeit wurden bei Expeditionen die ersten Verträge mit Stammesführern geschlossen und drei spanische Siedlungen gegründet. ${ }^{4}$ Das auf Betreiben einer "spanischen Afrika-Gesellschaft" ausgerufene "spanische Protektorat der afrikanischen Küste" für das Gebiet von Cap Blanc bis Cap Bojador wurde Spanien am 26. Februar 1885 auf der Berliner 'Konferenz über die Aufteilung Afrikas unter die europäischen Staaten ${ }^{5}$ zugeteilt. Die Versuche spanischer Truppen, dieses Gebiet zu halten, scheiterten am Widerstand der Bevölkerung. Nach heftigen Kämpfen 1887, 1892 und 1894 gelang es den Spaniern nur, einen schmalen Landstrich in Höhe des heutigen Dakhla (Villa Cisneros) zu halten. Zu dieser Zeit trat die spanische Regierung mit Frankreich in Geheimverhandlungen ein, um den Grenzverlauf zum französischen Einflußbereich (Algerien, Marokko, Mauretanien) festzulegen. Mit den Konventionen vom 27. Juni 1900, vom 8. April 1904 und vom 27. November 1912 entstanden die Kolonialgrenzen der Westsahara. ${ }^{6}$ In diesen Verträgen verzichtete Frankreich zugunsten von Spanien auf das zunächst von ihm gehaltene, etwa 26000 qkm große Protektorat Spanisch-Südmarokko, das Gebiet von Tarfaya. Spanien versuchte, in der Westsahara weitere Militärstützpunkte zu errichten. Der Widerstand der Bevölkerung unter der Führung der 'Blauen Sultaner richtete sich nicht nur gegen Spanien und war nicht auf das Gebiet der Westsahara beschränkt. Bekämpft wurde mit sahraouischer Unterstützung auch Frankreichs Kolonialherrschaft über Marokko und sein Versuch, Mauretanien zu erobern. Wegen der Stärke des Widerstands gegen die Kolonialmächte und des als zu gering empfundenen spanischen militärischen Engagements, drohte Frankreich Spanien, die Westsahara zu besetzen. Spaniens zunehmenden militärischen Anstrengungen konnten

3 Zur Frühgeschichte Rumeu de Armas, Antecedentes históricos del Sahara Español, in: Africa (Madrid), (1970)346, S. 4-10.

4 Vgl. Thompson/Adloff, The Western Saharans, Backgrounds to conflict, London 1980, S. $104 \mathrm{ff}$.; siehe auch Clausen, Zur politische Zukunft der Spanischen Sahara, in: Orient, (1973)2, S. 79-82(80).

5 Vgl. Miské, front polisario - l'âme d'un peuple, Paris 1978, S. 83 ff.

6 Vgl. Barbier, Le conflit du Sahara Occidental, Paris 1982, S. 57ff.; Rézette, The Western Sahara and the frontiers of Morocco, Paris 1975, S. 64ff. und Lazrak, Le contentieux territorial entre le Maroc et l'Espagne, Casablanca 1975, S. 73ff., der teilweise andere Daten nennt. 
nicht verhindern, daß ab 1952 eine auch vom Gebiet der Westsahara aus operierende Befreiungsarmee die Unabhängigkeit Marokkos erkämpfte. Die zahlreichen Sahraouis in dieser Armee beteiligten sich später auch am Unabhängigkeitskampf Algeriens.

Die sahraouische Beteiligung an den antikolonialen Befreiungskämpfen in Marokko und Algerien war das erste deutliche Anzeichen eines "modernen" Antikolonialismus in der Sahara, der in der Folgezeit auch zur Herausbildung von sahraouischen Befreiungsorganisationen führte.

Das seit 1956 unabhängige Marokko unter König Mohammed V. war nicht bereit, sich seinerseits am Unabhängigkeitskampf der Sahraouis gegen Spanien zu beteiligen. Im Gegenteil lieferte Marokko auf seiner Seite kämpfende Sahraouis an Spanien aus und erhob eigene Ansprüche auf ein "Großmarokko". Nach den Vorstellungen des in dieser Frage mit dem König konformgehenden einflußreichen Präsidenten der Istiqlal-Partei, Allal El Fassi, sollte dieses Großreich neben Marokko die spanischen Presidios in Nordmarokko, die Enklave Ifni, die Westsahara, ganz Mauretanien, den Norden der Republik Mali und die südwestliche algerische Sahara umfassen. ${ }^{7}$

Marokko erhielt 1958 von Spanien das geologisch zur Sahara gehörende Gebiet von Tarfaya. Die Westsahara wurde 1958 offiziell zur spanischen Kolonie erklärt und 1970 als Region nach Spanien eingegliedert. Hiergegen gerichtete Demonstrationen in der Hauptstadt Al-Ayoun wurden blutig aufgelöst. ${ }^{8}$ Marokko und Mauretanien schlossen einen Beistands- und Nachbarschaftsvertrag und bekundeten gemeinsam mit Algerien die Absicht, die "Dekolonialisierung der spanischen Sahara auf der Basis der Resolution der Vereinten Nationen nun schnell voranzubringen". Ihr "unaufhebbares Festhalten an den Prinzipien der Selbstbestimmung" bekräftigten die drei Staatschefs dieser Staaten anläßlich eines Treffens 1973. Spanien hielt an seiner Präsenz in der Westsahara fest, schlug aber die Gewährleistung innerer Autonomie der Westsahara vor. In der Westsahara ging aus einer Anzahl von Befreiungsbewegungen, insbesondere der ersten größeren antikolonialen Partei MLS, die am 10. Mai 1973 gegründete Frente POLISARIO (Volksfront für die Befreiung von Saguia El Hamra und Rio de Oro) hervor.

Die F. POLISARIO stand in der Tradition der vorangegangenen Befreiungsbewegungen und wurde nach ihrer Gründung binnen weniger Monate zur dominierenden Kraft in der Sahara.$^{8 a}$ Zur gleichen Zeit verkündete die in innenpolitischer Bedrängnis stehende spanische Regierung, daß sie die Souveränität über die Sahara so schnell wie möglich übergeben und unter Umständen auf das Referendum aus "nicht in ihrer Macht liegenden Gründen" verzichten wolle.

Mit seinen propagandistischen Vorbereitungen für einen "Grünen Marsch" in die

7 Vgl. Miské, a.a.O. (Anm. 5), S. 51 ff. und Thompson/Adloff, a.a.O. (Anm. 4), S. 222.

8 Vgl. Clausen, Der Konflikt um die Westsahara, Hamburg 1978, S. 37.

8a Zur Geschichte der Befreiungsbewegungen und der Organisation der sahraouischen Gesellschaft Barbier, L'avenir du Sahara Espagnol, in: Politique étrangère, 40(1975)4, S. 353-380(368ff.); Wexsteen, La question du Sahara occidental, in: Annuaire de l'Afrique du Nord, 15(1976), S. 255-275(256ff.) und Dessens, Le Litige du Sahara occidental, in: Maghreb-Machrek, (1976)71, S. 28-46(32f., 38f). 
Sahara, ${ }^{9}$ der Besetzung des Landes durch Marokkaner in Zivil, machte der Sohn von Mohammed V., König Hassan II., im August 1974 deutlich, daß er eine Unabhängigkeit der Sahara verhindern wollte. Er wies alle Bemühungen um ein Referendum über die Zukunft des Landes zurück. Marokkos Aufforderung an Algerien und Mauretanien, seine Ansprüche auf das Gebiet der Westsahara zu unterstützen, führten im Oktober $1974 \mathrm{zu}$ einem Geheimabkommen zwischen Marokko und Mauretanien über eine zukünftige Teilung der spanischen Sahara. Letztmalig schlug Spanien im Juli 1975 der Djemaa, einer von Spanien nach traditionellem Vorbild eingerichtete sahraouische Stammesvertretung, ein Referendum für 1976 vor. Die Djemaa ihrerseits emanzipierte sich 1975 von ihrem kolonialen Ziehvater und erklärte die F. POLISARIO in der 'Deklaration von Guelta zur legitimen Vertretung des sahraouischen Volks. Hassan kündigte in dieser Situation in öffentlicher Rede den "Einmarsch des marokkanischen Volks" in der Westsahara für 1975 an.

Am 6. 11. 1975 drangen 350000 Zivilisten aus Marokko unter Begleitung von mehreren tausend Soldaten in die Sahara ein. Spanien, das sich wegen des im Sterben liegenden Franco innenpolitisch in gespannter Situation befand, ließ den Marsch bis $15 \mathrm{~km}$ hinter die Grenze zu. Hassan II. sah seine Ziele erfüllt und rief die Marschierer nach Marokko unter Zurücklassung des nachgefolgten Militärs zurück. Spanien, Mauretanien und Marokko unterzeichneten am 14. 11. 1975 einen mehrere Wochen lang geheimgehaltenen 'Madrider Vertrag', nach dem Spanien die Westsahara 1976 verlassen sollte. Die letzten spanischen,Legionäre zogen bereits im Januar 1976 ab. Die Verwaltung dieses spanischen Kolonialgebiets war bereits zuvor vertragsgemäß an Marokko und Mauretanien übergeben worden. In die Garnisonen zogen die Streitkräfte der spanischen Vertragspartner ein. Mit der Ausreise der letzten Offiziellen am 26. 2.1976 endete die Kolonialzeit Spaniens über die Westsahara. Spanien hat sich damit der Verantwortung auf eine in der Kolonialgeschichte einzigartige Weise entzogen. In einem Schreiben an den Generalsekretär der Vereinten Nationen brachte Spanien zwar seine Ansicht zum Ausdruck, daß der Entkolonialisierungsprozeß nicht abgeschlossen sein würde, bevor nicht der Wille der Bevölkerung verläßlich zum Ausdruck gebracht werde. Die Chance, ihren Willen etwa in Form eines Referendums zu äußern, hatte Spanien der Bevölkerung aber genommen.

In der Nacht auf den 27. 2. 1976 wurde auf dem Gebiet der Westsahara die "Demokratische Arabische Republik Sahara" (DARS) proklamiert, deren erste Regierung am 4. 3. 1976 benannt wurde. Die Truppeneinmärsche und die Zunahme der Kämpfe zwischen Marokko und Mauretanien auf der einen, der sahraouischen Volksbefreiungsarmee (ALPS) auf der anderen Seite führten zu einer Massenflucht der sahraouischen Bevölkerung nach Algerien, wo die F. POLISARIO den Aufbau von Flüchtlingslagern mit Unterstützung Algeriens in der Wüste bei Tinduf organisierte. Die folgenden Jahre

9 Vgl. die unterschiedlichen Erklärungen des Marsches von Byman, The March on the Spanish Sahara; A Test of International Law, in: Journal of International Law and Policy, (1976), S. 95-121(112ff.) und Weiner, The green march in historical perspective, in: The Middle East Journal, 33(1979)1, S. 20-33. 
waren bestimmt durch heftige Guerilla-Kämpfe der ALPS, die in den letzten Jahren teilweise auch zu Angriffen in offenen Verbänden überging. Gleichzeitig bemühten sich die streitenden Parteien auf diplomatischer Ebene um eine Unterstützung ihrer jeweiligen Position.

Nach dem Sturz der Regierung Ould Dadda in Mauretanien am 10. Juli 1978 rief die F. POLISARIO einseitig einen Waffenstillstand aus. Er war die Vorstufe zu einem im August 1979 abgeschlossenen Friedensvertrag, in dem Mauretanien auf jeden Gebietsanspruch verzichtete. Nach dem Rückzug Mauretaniens aus dem Südteil der Westsahara schloß Marokko auch diese Provinz seinem Staatsgebiet an.

1980 hat Marokko nach einer Serie von militärischen Niederlagen damit begonnen, einen Verteidigungswall aus Mauern, Zäunen, Sandwällen, Gräben und Minenfeldern durch die Sahara zu ziehen, um die eigenen Garnisonen vor der F. POLISARIO besser zu schützen und in diesem gesicherten Bereich mit der Ansiedlung von zugewanderten Marokkanern zu beginnen. In diesem sog. "nützlichen Dreieck" sollte mit dem Abbau von Bodenschätzen begonnen werden. Der Wall zieht sich auf sahraouischem Gebiet von der Atlantikküste in nordöstliche Richtung bis tief in das marokkanische Landesinnere hinein. Das Gebiet außerhalb der Wallanlagen wird uneingeschränkt von der F. POLISARIO kontrolliert. Lediglich in Dakhla im Süden gibt es einen marokkanischen Außenposten, der aber wie die nördlichen Garnisonen im 'nützlichen Dreieck` und in Südmarokko ständig mit Angriffen rechnen muß.

\section{Konfliktbeteiligte Staaten}

\subsection{Spanien}

Die spanische Kolonialpolitik trägt die Hauptverantwortung für die Entstehung des Westsahara-Kriegs. ${ }^{10}$ Nachdem 1947 bei Al Ayoun in der Sahara Phosphatlager entdeckt wurden, engagierte sich Spanien stärker in der Westsahara, konnte aus Geldmangel die Prospektierung aber in den folgenden 15 Jahren nicht beginnen. Das minderte zunächst die Aussicht, sich in die Phalanx der wichtigsten Phosphatproduzenten der Welt mit den USA, Marokko und der Sowjetunion einreihen zu können. Erst 1972 konnte die staatseigene Gesellschaft 'Fosfatos de Bou-Craa' mit ausländischer Finanzhilfe 1972 den Phosphatabbau bei Bou-Craa, hundert Kilometer von Al Ayoun entfernt, beginnen. ${ }^{11}$ Spaniens Interesse an der Westsahara beschränkte sich im übrigen weitgehend auf die Errichtung von Militärbasen und auf die Ausbeutung der Ressourcen

10 Vgl. Ortiz de la Torre, Sahara Occidental, "Terra Nullius«?, in: Revista General de Legislacion y Jurisprudencia, 124(1975)6, S. 563-605(602f.).

11 Vgl. Weinstein, The Western Sahara, in: Current History, 78(1980)455, S. 110-114 und 136-137(113); Lippert, Emergence or submergence of a potential State: The struggle in Western Sahara, in: Africa today, (1977)1, S. 41-60(48f.). 
des Landes. Gretton weist zu Recht darauf hin, daß die Jahre der spanischen Mißherrschaft eine Situation geschaffen haben, in der es außerordentlich schwer für eine unabhängige Westsahara wäre, als moderner Staat autonom zu existieren. ${ }^{12}$ Im Gegensatz zu anderen Kolonialmächten unternahm Spanien auch keine Anstrengungen, eine nationale sahraouische Elite auszubilden, etwa um sich eine spätere nationale Regierung gewogen zu halten. Die auf eine Dekolonialisierung der Spanischen Sahara drängenden Internationalen Organisationen veranlaßten Spanien, der Forderung nach einem Referendum zuzustimmen, mit dem das sahraouische Volk sein Recht auf Selbstbestimmung ausüben sollte. ${ }^{13}$ Faktisch tat Spanien allerdings alles, um das Referendum hinauszuzögern. ${ }^{14}$ Es lud Marokko und Mauretanien nach Madrid zu einer Drei-Mächte-Konferenz, an der Algerien auf marokkanischen Wunsch nicht beteiligt wurde. Die Vertreter der drei Staaten schlossen am 14. 11. 1975 den bereits erwähnten 'Madrider Vertrag'. Spanien erhoffte sich davon das Wohlverhalten wichtiger arabischer Staaten als Handelspartner, ein Ruhen der marokkanischen Ansprüche auf die Presidios Ceuta und Melilla, die Sicherung der wirtschaftlichen Interessen in der Sahara und die Verhinderung der Unterstützung der Separatisten-Bewegung auf den Kanarischen Inseln durch eine souveräne Sahara. Durch den fait accompli waren alle Hoffnungen auf eine Ubergabe des Gebiets an die F. POLISARIO, die von der sahraouischen Bevölkerung als ihre Vertretung anerkannt wurde, ${ }^{15}$ zerschlagen. Diese waren aufgekommen, nachdem die Djemaa selbständig die Einrichtung einer spanisch-sahraouischen Kommission beschlossen hatte, die die Souveränitätsübergabe überwachen und später als 1. Exekutive des Landes fungieren sollte. Auch der spanische Außenminister und der Generalsekretär der F. POLISARIO hatten sich getroffen und in vielen Punkten bereits Einigung über die Úbergabemodalitäten erzielt. ${ }^{16}$

Der Madrider Vertrag sah vor, daß sich Spanien bis zum 28. 2. 1976 schrittweise aus der Sahara zurückziehen und die Verwaltung unverzüglich an die Vertragsparteien übergeben sollte. Spanien erhielt für seinen Verzicht die Zusicherung der Rückzahlung aller Investitionen im Phosphat-Abbaugebiet, eine 45 \%ige Beteiligung an der Prospektionsgesellschaft, die Garantie einer Deckung spanischer Phosphatbedürfnisse, die Sicherung von Fischereirechten, die Stillhaltezusicherung Marokkos in der Frage der Rückforderung von Ceuta und Melilla. ${ }^{17}$

Damit war die Teilung der Westsahara zwischen Marokko und Mauretanien faktisch vorbestimmt. Diese beiden Staaten legten dann im April 1976 die Grenze entsprechend

12 Western Sahara - The fight for Self-Determination, London 1976, S. 29.

13 Ausführlich Franck, The stealing of the Sahara, in: American Journal of International Law, 70(1976), S. 694-721(703ff.).

14 Vgl. Clausen, Der Konflikt um die Spanische Sahara, in: Orient, 16(1975)4, S. 21-38(28f.) und Gretton, a.a.O. (Anm. 12), S. $27 \mathrm{ff}$.

$15 \mathrm{Vgl}$. Report of Visiting Mission to Spanish Sahara (UN), 1975, A/10023/Add. 5, 7. 11. 1975.

$16 \mathrm{Vgl}$. Thompson/Adloff, a.a.O. (Anm. 4), S. 132ff. und Le monde 2. 12. 1975.

17 Vgl. Kühlein, Der Entkolonialisierungsprozeß der Spanischen Sahara, in: Vereinte Nationen, 23(1975)6, S. 169-174 und 24(1976)1, S. 25(25) und Clausen, a.a.O. (Anm. 8), S. 59f. 
ihrem zweiseitigen Vertrag vom Oktober 1974 - geschlossen zu einem Zeitpunkt, als noch alle offiziellen Erklärungen auf ein Referendum abzielten - fest. Durch die Lobby Marokkos in der spanischen Regierung ${ }^{18}$ veranlaßt und durch den herannahenden Tod Francos innenpolitische Schwierigkeiten fürchtend, auf die es sich zu konzentrieren galt, verabschiedete die Cortes am 18. 11. 1975, zwei Tage vor Francos Tod das Gesetz über die Entkolonialisierung, ohne allerdings den vollen Wortlaut der Vereinbarung überhaupt zu kennen. Erst im Dezember wurde der Wortlaut des "Madrider Vertrags" bekannt. ${ }^{19}$ Die bedeutungsvollen Zusatzabkommen sind nur teilweise öf fentlich bekannt geworden. ${ }^{20}$

Die Diskussion um das Madrider Abkommen war damit aber nicht beendet. Das zeigten auch die am 10.12. 1975 in den UN verabschiedeten Resolutionen. ${ }^{21}$ Allein die von Marokko unterstützte Resolution nahm den Vertrag überhaupt zur Kenntnis. Spanien beteuerte, daß es nicht die Souveränität an Marokko und Mauretanien, sondern nur die verwaltungsmäßige Herrschaft übertragen habe.

In den folgenden Jahren forderte die damals oppositionelle sozialistische Partei (PSOE), unterstützt durch die Kommunisten (PCE), immer wieder, vom Vertrag zurückzutreten bzw. ihn für nichtig zu erklären. Spaniens Regierungspräsident Adolfo Suarez antwortete darauf: "España no es responsable de lo que sucede en el Sahara Occidental actualmente. Sus responsabilidades cesaron tras la cesión de poderes a Marruecos y Mauritania y la retirada del territorio. ${ }^{22}$

In einer Parlamentsdebatte über einen Fischereivertrag mit Marokko fragte die Opposition 1978 noch einmal an, "weshalb das Abtretungsabkommen entgegen den Gesetzen nie veröffentlicht, geschweige denn, mit seinen Zusatzklauseln zur Ratifizierung vorgelegt worden sei.“"23 Im Februar 1978 stimmte die Cortes schließlich mit 142:175 Stimmen gegen die Widerrufung des Madrider Vertrags. ${ }^{24}$

Nach dem Dekolonisierungsrecht wäre die Kolonialmacht Spanien im übrigen verpflichtet gewesen, in ihrer Sahara-Kolonie einen Dekolonisierungsprozeß einzuleiten. Wenn auch die Modalitäten dieses Prozesses nicht im einzelnen festgelegt sind, so ist doch anerkannt, daß diese Rechtspflicht die Ausübung des Selbstbestimmungsrechts durch die Bevölkerung in der Kolonie beachten muß. ${ }^{25}$ Die Vereinten Nationen haben die

$18 \mathrm{Vgl}$. die aufschlußreichen Hinweise bei Clausen, Spanien und die Maghreb-Staaten, in: Orient, 21(1980)1, S. 18-41(30f.) und dies., a.a.O. (Anm. 14), S. 33f.

19 In deutscher Ubersetzung bei Clausen, a.a.O. (Anm. 8); Alberich, España y el Sah ara Occidental. Antecedentes de una descolonización, in: Revista de Politica Internacional, (1977)154, S. 9-52(48f.) und Ortiz de la Torre, Recientes aspectos juridicointernacionales del conflicto del Sahara, in: Revista General de Legislación y Jurisprudencia, 126(1977)6, S. 555-590; vgl. auch Miguez, Le Sahara Occidental et la politique maghrébine de l'Espagne, in: Politique étrangère, 43(1978)2, S. 173-180.

20 Veröf entlicht von Ortiz de la Torre, a.a.O. (Anm. 19), S. 563.

21 General Assembly Res. 3458A und 3458B(XXX).

22 Zit. bei Cola Alberich, a.a.O. (Anm. 19), S. 9.

23 Kassebeer, Eine neue Rollenverteilung im Maghreb, in: Süddeutsche Zeitung vom 18. 2. 1978, S. 7.

24 Eine Rechtf ertigung der spanischen Saharapolitik findet sich bei Cola Alberich, a.a.O. (Anm. 19), passim.

25 Vgl. Kunig, Das völkerrechtliche Nichteinmischungsprinzip, Baden-Baden 1981, S. 367. 
Selbstbestimmungsformel in Art. 1 Ziffer 2 und in Art. 55 der Charta aufgenommen. Art. 1 Ziffer 2 beschreibt als eines der grundlegenden Ziele der Vereinten Nationen, "to develop friendly relations among nations based on respect for the principle of equal rights and self-determination of peoples, and to take other appropriate measures to strengthen universal peace". Art. 1 Ziff. 3 konkretisierend, zählt Art. 55 die im Rahmen der internationalen wirtschaftlichen und sozialen Zusammenarbeit zu verfolgenden Ziele mit der Einleitung auf:

"With a view to the creation of conditions of stability and well-being which are necessary for peaceful and friendly relations among nations based on respect for the principle of equal rights and self-determination of peoples, the United Nations shall promote: ..." Die Aufnahme des Begriffs vom Selbstbestimmungsrecht in die UN-Charta hat keineswegs auch die Frage nach seiner Rechtsnatur beantwortet. Gestritten wird in der Völkerrechtswissenschaft insbesondere, ob das Selbstbestimmungsrecht ein moralisches Prinzip oder politisches Programm ist, ob es durch Aufnahme in die Satzung zur Rechtsnorm des positiven Völkervertragsrechts geworden ist, die die Rechte und Pflichten der Mitglieder der Vereinten Nationen oder gar aller Völkerrechtssubjekte statuiert, ob es als allgemeiner Grundsatz oder als Teil des Gewohnheitsrechts - ohne sich zur Norm verdichtet zu haben - völkerrechtliche Bedeutung hat und ob es sich auf die Völker aus den Kolonien oder Kolonien beschränkt oder von Volksteilen und zu jedem Zeitpunkt beansprucht werden kann. Bedeutsam ist im vorliegenden Fall, daß alle an der Lösung des Konflikts interessierten Parteien gemeinsam davon ausgehen, daß die Bewohner der ehemaligen Kolonie 'Spanisch Sahara das Recht haben, über ihre Zukunft selbst zu entscheiden.

Das hat auch Marokko gefordert, als Spanien Kolonialherr über die Sahara war; ${ }^{26} 1981$ hat es dies wieder durch eigene Vorschläge für ein Referendum und die Akzeptierung der von der OAU vorgeschlagenen Fragestellung anerkannt. Algerien ist kontinuierlich für das Selbstbestimmungsrecht der Sahraoui eingetreten, was sich in der Initiierung von Resolutionen in den UN und der OAU zeigt. Mauretanien hat sich ebenfalls zu einem Verfechter dieses Rechts in der OAU entwickelt. Selbst Spanien hatte ein Referendum zur Gewährleistung des Selbstbestimmungsrechts vorbereitet und in Auslegung des Madrider Abkommens darauf hingewiesen, daß nur die vorübergehende Verwaltung an Marokko und Mauretanien übertragen wurde und die Wahrnehmung des Rechts auf Selbstbestimmung in der ehemaligen Kolonie noch ausstehe. Die F. POLISARIO reklamiert zwar für sich, daß das Volk der Sahara durch die Gründung der Demokratischen Arabischen Republik Sahara bereits ihren Willen zum Ausdruck gebracht hat, zeigt sich aber mit der Durchführung eines freien Referendums einverstanden.

Anwendung findet im Fall der Westsahara die UN-rechtliche Legaldefinition vom Selbstbestimmungsrecht der Völker, wie sie anläßlich des 25. Jahrestags der UNO in der

26 Vgl. Ortiz de la Torre, a.a.O. (Anm. 10), S. 603 und Carillo Salcedo, Momento internacional: Problemas juridicos en presencia en la cuestión del Sahara, in: Revista de Derecho Público (1975)57, S. 35. 
"Erklärung über Völkerrechtsgrundsätze betr. die freundschaftlichen Beziehungen und die Zusammenarbeit zwischen Staaten gemäß der Satzung" gegeben wurde. Diese Erklärung umfaßt sieben Grundsätze. Punkt sechs beschreibt die besondere Rechtsstellung der Kolonien, "bis das Volk sein Selbstbestimmungsrecht gemäß der Satzung und insbesondere ihrer Grund- und Zielbestimmungen ausgeübt hat". Danach besitzt die Bevölkerung einer Kolonie die tatsächliche und rechtliche Möglichkeit, über die Gestaltung der Zukunft eine freie Entscheidung zu treffen. ${ }^{27}$ Heranzuziehen ist auch die "Erklärung über die Gewährung der Unabhängigkeit an koloniale Länder und Völker", die Resolution 1514(XV) vom 14. 12. 1960. Die Ausführungen der UN verdeutlichen, daß die Unterwerfung von Völkern unter fremde Oberhoheit und Ausbeutung eine satzungswidrige Mißachtung des (kollektiven) Selbstbestimmungsrechts und der (individuellen) Menschenrechte und Grundfreiheiten darstellt. ${ }^{28}$ Jegliche, auf die Unterdrückung von Selbstbestimmungsansprüchen gerichtete Gewaltanwendung ist untersagt. Der Dekolonialisierungsprozeß war, als Spanien sich aus der Sahara zurückzog, jedenfalls nicht abgeschlossen. Der Madrider Vertrag kann daher nicht die von Marokko gewünschte Wirkung entfalten. Er kann nicht den Abschluß einer rechtmäßigen Dekolonialisierung und damit eine Erledigung des Selbstbestimmungsanspruchs begründen. Diese Einschätzung der völkerrechtlichen Qualität des Vertrags unterstreicht die folgende Auffassung: Marokko - und zunächst auch Mauretanien - ist an die Stelle Spaniens als in dem Gebiet die Gewalt ausübende Macht getreten. Als solche muß sie sich die Ansprüche der Bevölkerung aus der ehemaligen Spanisch-Sahara entgegenhalten lassen, die sie aus dem Dekolonisierungsrecht herleiten kann. Das saharaouische Volk kann demnach sein Recht auf Widerstand, das völkerrechtlich zugestandene Recht auf Abweisung einer Unterdrückung, nunmehr gegen die Aggression und Okkupation Marokkos geltend machen. Spanien hätte dem sahraouischen Volk die Möglichkeit eröffnen müssen, frei darüber zu entscheiden, ob ein eigener Staat gegründet, eine Integration in einen bestehenden oder die Assoziierung mit einem Staat erfolgen soll. ${ }^{29}$ Dieses ist im übrigen eine Verpflichtung, mit der diejenige dritter Staaten - wie Marokko und Mauretanien - korrespondiert, die das Selbstbestimmungsrecht beachtenden Dekolonisierungsmaßnahmen nicht zu stören. Auch Kunig hält den spanischen Dekolonisierungsprozeß in der Sahara für rechtswidrig, weil die Uberlassung der früheren Kolonie 'Spanisch-Sahara' an Marokko und Mauretanien durch den 'Madrider Vertrag' nicht den geltenden Dekolonisierungsnormen entsprach. ${ }^{30}$

27 Im Kontext der Dekolonisierung ist das Prinzip des Selbstbestimmungsrechts am stärksten herausgearbeitet; vgl. dazu auch Kunig, a.a.O. (Anm. 25), S. 369.

28 Vgl. Rabl, Das Selbstbestimmungsrecht der Völker, Köln Wien ${ }^{2} 1973$, S. 492.

$29 \mathrm{Vgl}$. Zourek, La lutte d'un peuple en vue de faire prevaloir son droit à l'autodétermination constitue-t-elle au regard du droit international un conflit interne ou un conflit de caractère international?, in: Studi in onore di Manlio Udina, Tomo I, 1975, S. 895 ff. (907).

30 A.a.O. (Anm. 25), S. 367f. 
Diese Auffassung düfte auch die Sozialistische Partei Spaniens (PSOE) 1976 als Oppositionspartei bewogen haben, durch Felipe González in der Westsahara gemeinsam mit der F. POLISARIO ein Dokument zu unterzeichnen, in dem das Dreierabkommen von Madrid für 'null und nichtig` erklärt und die drei vertragschließenden Parteien verurteilt wurden. Nach der Regierungsübernahme durch die Sozialisten hat sich die spanische Regierungspolitik gegenüber Marokko und Spanien allerdings kaum geändert. Nach den Besuchen des Außenministers, des Vizepräsidenten und Präsidenten 1982/83 in Marokko wurde die promarokkanische Außenpolitik auch durch die neue sozialistische Regierung deutlich. Die Normalisierung der Beziehung zu Marokko, der Abschluß eines Fischereiabkommens und die Bemühungen um eine marokkanische Zusage, die spanischen Enklaven in nächster Zeit nicht zurückzufordern, haben Vorrang vor der Aufrechterhaltung der traditionellen Freundschaft zwischen der PSOE und der F. POLISARIO. Die spanische sozialistische Regierung hat erklärt, daß es auch weiterhin nicht bereit sei, das Abkommen zu kündigen, während der Vorstand der PSOE der F. POLISARIO in einer Botschaft zum 8. Jahrestag des Madrider Abkommens am 14. November 1983 seine "Unterstützung für den Kampf des sahraouischen Volks" bekräftigte.

\subsection{Marokko}

Marokko war gerade unabhängig geworden, als der Führer der bürgerlich-konservativen Istiqlal-Partei, Allal El-Fassi, im Jahre 1956 unter Berufung auf nationale, historische, geografische und soziale Einheit im Benehmen mit dem Herrscherhaus Ansprüche auf ein "großmarokkanisches Reich" erhob, das die spanischen Presidios, die Enklave Ifni, Spanisch-Südmarokko, die Spanische Sahara, Mauretanien, Teile Malis und Algeriens mit umfassen sollte. ${ }^{31}$ Spanien einigte sich 1969 mit Marokko über die Rückgabe der Enklave Ifni. Die Forderung der Rückgabe der spanischen Presidios stellte Marokko zunächst zurück. Auf die übrigen Gebiete, mit Ausnahme der Sahara, verzichtete das Königreich, nachdem mehrere Grenzverträge mit Algerien geschlossen und das Ministerium für mauretanische Angelegenheiten aufgelöst ${ }^{32}$ worden war.

Im Frühjahr 1974 verkündete Hassan II. seinen Willen, die besetzte Sahara mit Marokko "wiederzuvereinigen". Die Massenmedien begannen, in der Bevölkerung eine nationalistische Bewegung zur Integration der Sahara zu entfachen. Die Gefahr eines Kriegs mit Spanien, das weiterhin ein Referendum forderte, wendete Hassan II. durch den diplomatischen Vorschlag ab, den Internationalen Gerichtshof in Den Haag mit der Westsahara zu befassen. Auf marokkanischen Antrag hin beauftragten die UN am 13. 12. 1974 den Gerichtshof mit der Erstellung eines (unverbindlichen) Gutachtens (advisory opinion). Durch diesen Schachzug hatte Marokko die notwendige Zeit gewon-

31 Vgl. Miské, a.a.O. (Anm. 5), S. 51 f.; Weiner, a.a.O. (Anm. 9), S. 22; Clausen, a.a.O. (Anm. 8), S. 29 und Franck, a.a.O. (Anm. 13), S. 694.

32 Vgl. Okere, The Western Sahara Case, in: The International and Comparative Law Quarterly, 29(1979), S. 296-312(297). 
nen, um das Abkommen mit Spanien auszuhandeln und den Teilungsvertrag mit Mauretanien zu schließen. ${ }^{33}$

Bei der Suche nach Handlungsmotiven, die zur unnachgiebigen Haltung Marokkos in der Westsaharafrage, zur Verweigerung von direkten Verhandlungen mit der F. POLISARIO und letztlich trotz Unterstützung weniger afrikanischer und westlicher Staaten in die politische Isolation führten, sind zwei Gründe herauszuheben. Zum einen ließ die schwierige wirtschaftliche Lage Marokkos ein schnelles Handeln Anfang der siebziger Jahre im Hinblick auf die zu erwartende Konkurrenz durch einen spanischen Phosphatabbau in der Sahara geraten erscheinen. Nach einem spanischen Rückzug mußte die Konkurrenz eines souveränen Saharastaats befürchtet werden. Bereits ohne die Vorkommen in der Westsahara besitzt Marokko 60 \% der Weltreserven an Phosphat und ist vor den USA sogar der größte Exporteur dieses auch als Düngemittel verwendeten Rohstoffs. Phosphat ist Marokkos wichtigste Devisenquelle. ${ }^{34}$ Die Kontrolle über die Lagerstätten in der Sahara soll die enormen Reserven am Phosphat sichern, insbesondere aber einen den Preis auf dem Weltmarkt beeinflussenden Konkurrenten verhindern. Die Krisen auf dem Phosphatmarkt 1973/74 und die damit verbundenen verheerenden Auswirkungen auf die Wirtschaft Marokkos verdeutlichen die wirtschaftliche Bedeutung dieses Rohstoffs. Neuerdings verfolgt Marokko konkrete Schritte, um mit Hilfe von US-Firmen aus Phosphat Uran zu gewinnen und sich damit eine neue Einnahmequelle zu sichern, die die hohen Kriegskosten von täglich etwa 1 Million US-Dollar mit finanzieren sollen.

Hassan II. ist auf die Sahara nicht nur ökonomisch, sondern auch politisch angewiesen. Mit der Sahara-Frage gelang es dem marokkanischen Herrscher immer wieder, alle politischen Kräfte des Volks über alle inneren Gegensätze und Spannungen hinweg zu solidarisieren. Die innenpolitischen Schwierigkeiten kamen durch zwei Staatsstreichversuche 1971 und 1972 durch Militärs zum Ausdruck, bei denen Hassan nur mit viel Glück dem Tod entging. ${ }^{35}$ Seine scharfen Polizeiverfolgungen, die Parteien- und Presseverbote, die Wahlfälschungen und die Aufhebungen des parlamentarischen Lebens brachten den Thron immer wieder ins Wanken. ${ }^{36} \mathrm{Da}$ die Unruhen im militärischen und administrativen Bereich nach wie vor anhalten, zeigt die Beseitigung des Generals Dlimi, dem mächtigsten Mann in Marokko nach dem König, im Januar 1983. ${ }^{37}$ In der Folgezeit führte Hassan zahlreiche Umbesetzungen beim Militär und in den Sicherheitsorganen

33 Vgl. Franck, a.a.O. (Anm. 13), S. $705 f f$.

34 Vgl. Byman, a.a.O. (Anm. 9), S. 96 und Gretton, a.a.O. (Anm. 12), S. 23.

35 Die Attentate und ihre Hintergründe beschreibt Tibi, Die ex-koloniale Armee: Marokko, in: ders., Militär und Sozialismus in der 3. Welt, Frankfurt/M. 1973, S. 138ff.

36 Vgl. Hottinger, Die Sahara-Frage, ein Krebsgeschwür in Nordafrika, in: Europa-Archiv, 34(1979)21, S. 653-661(653).

37 Dlimi war 1965 in Paris an der Ermordung des marokkanischen Oppositionspolitikers Ben Barka beteiligt, vgl. Scholl-Latour, Allah ist mit den Standhaften, Stuttgart 1983, \$. 61. Zuletzt war er der für die Kriegführung in der Sahara verantwortliche Kommandeur der Militärregion Süd und gleichzeitig Geheimdienstchef, verantwortlich für die Gegenaufklärung und Leiter der Königlichen Adjudantur. Zur Beseitigung Dlimis Herzog, in FR 14. 2. 1983. 
durch. Der Feldzug des Monarchen zur "Repatrisierung" der Sahara konnte den inneren Frieden aufgrund der Verschärfung der sozialen Lage kaum noch aufrechterhalten. Auch die Neue Züricher Zeitung stellte fest, "daß der Westsahara-Krieg seine Funktion, das Volk ans Regime zu binden, nicht mehr erfüllt “. ${ }^{38}$ Hintergrund dieser Feststellungen sind die Aufstände im Juni und Juli 1983 in zahlreichen marokkanischen Städten, insbesondere in der 3 Millionen Einwohner zählenden Stadt Casablanca. ${ }^{39}$ Die Unruhen im Anschluß an Preiserhöhungen der Grundnahrungsmittel bis zu $85 \%$ forderten regierungsoffiziell 66 Tote, während andere Quellen von 1000 Toten allein in Casablanca sprechen. ${ }^{40}$

Marokko steckt nach wie vor in einer tiefen Wirtschaftskrise. Zwar wurden die vorgenannten Preiserhöhungen um 50 \% zurückgenommen, doch liegen die Preise für Zucker, Ol, Milch, Mehl und Butter immer noch um 40-60\% über den vormaligen. ${ }^{41}$ Für die marokkanische Bevölkerung, die nach einem Bericht der Weltbank von Ende $1979 \mathrm{zu}$ einem Drittel unter der absoluten Armutsgrenze lebt und deren Situation sich nach einer katastrophalen Dürre noch weiter verschlechtert hatte, sind aber auch diese Erhöhungen nicht zu verkraften, insbesondere nicht von der extrem hohen Zahl arbeitsloser Marokkaner. Die Preissteigerungen der Grundnahrungsmittel waren für notwendig gehalten worden, weil der internationale Weltwährungsfond eine Aufgabe der marokkanischen Subventionspolitik als Voraussetzung für die Gewährung eines Kredits der Weltbank in Höhe von 87 Millionen Dollar verlangte. ${ }^{42}$ Neben einer 40 \%igen Haushaltsbelastung durch Kriegskosten ${ }^{43}$ ist Marokko zudem gezwungen, einen Großteil des Budgets für Olund Getreideimporte auszugeben. Die wirtschaftspolitischen Schwierigkeiten zeigen sich auch im 40 \%igen Kaufkraftschwund. ${ }^{44}$ Marokkos Hoffnungen auf eine Belebung der Wirtschaft durch den Wirtschaftskooperationsvertrag mit den USA und durch ein Gesetz, das eine Offnung des Binnenmarkts für ausländisches Kapital ermöglichen soll, ${ }^{45}$ haben sich nicht erfüllt. Die amerikanischen Geldgeber und Waffenimporteure drängen vielmehr derzeit auf Begleichung der Rechnungen. ${ }^{46}$

Innenpolitisch nimmt Hassan II. nach der Liquidierung des Generals Dlimi und anderer Militärs sowie nach Umbesetzungen in der Administration ${ }^{47}$ weiterhin verfassungsüberschreitenden Einflußr auf das parlamentarische Leben: Die Ergebnisse der Kommunalwahlen vom Juni 1983 wurden als der größte der vielen Wahlschwindel kommentiert, die

38 Neue Zürcher Zeitung (NZZ) 24. 6. 1981; ebenso El País 7. 7. 1981.

39 Frankfurter Allgemeine Zeitung (FAZ) 24. 6. 1981; NZZ 8. 8. 1981.

40 El País 7.7. 1981; FAZ 13. 7. 1981.

41 NZZ 24. 6. 1981: Le matin du Sahara 4.6. 1981.

42 Le monde 4. 6. 1981; zur desolaten sozialen Situation Selhami, Sécheresse: seule responsable?, in: jeune af rique, No. 1054 vom 18. 3. 1981, S. 36f.

43 FAZ 24. 6. 1981.

44 El País 7. 7. 1981.

45 Die dadurch einsetzenden Aktivitäten amerikanischen Kapitals werden beschrieben in: Af rica Now, (1982)8.

46 Süddeutsche Zeitung (SZ) 10.6. 1983.

47 Vgl. Archiv der Gegenwart vom 25. 1. 1982 (25282B) und afrique-asie, No. 294 vom 25. 4. 1983. 
es bisher in Marokko gegeben habe. ${ }^{48}$ Die für den Herbst 1983 vorgesehenen Parlamentswahlen wurden vom König ausgesetzt und mit der Begründung auf unbestimmte Zeit verschoben, daß zunächst das Referendum "zur Wiedererlangung unserer usurpierten Sahara" alle Kraft des Volks erfordere..$^{49}$ Im Oktober 1983 ließ sich der Monarch vom Parlament alle legislativen Vollmachten übertragen, während das im November von ihm neu gebildete Kabinett keine Ressortverteilung kennt und keine Befugnisse hat, sondern durch die Beteiligung aller Parteien den nationalen Konsens in ökonomisch und innenpolitisch gespannter Lage nach außen glaubhaft machen soll..$^{50}$

Der absolutistische Herrscher wies darauf hin, daß das Referendum die Zugehörigkeit der Sahara "zum marokkanischen Vaterland" bestätigen müsse. ${ }^{51}$ Nachdenklich stimmt sein Hinweis, daß in dem "undenkbaren" Fall, daß das Referendum negativ für Marokko ausfalle, niemand Marokko verpflichten können, "die Sahara zusammen mit den dort lebenden Marokkanern einer Bande von Söldnern auf einem Silberteller zu überreichen. “52 Ein den Unabhängigkeitswunsch ausdrückendes Referendumsergebnis würde von Marokko offensichtlich nicht anerkannt werden. Wohlweislich verschwieg Hassan in seiner Rede, daß sich eine marokkanische Delegation im April 1983 zu direkten Verhandlungen mit Regierungsvertretern der Republik Sahara, die offiziell kategorisch abgelehnt werden, getroffen hatte. Auf marokkanischer Seite nahmen an dem Treffen der Vertraute des Königs, Rueda Guerida, Außenminister Boucetta sowie der Innenminister Basri, auf sahraouischer Seite unter der Leitung des Premierministers Lamin der Ministerpräsident Ali Beiba und der für Außenbeziehungen verantwortliche Salem Ould Salek teil. Wie in Nairobi im Juni 1983 bekannt wurde, lehnte die Republik Sahara den marokkanischen Verhandlungsvorschlag ab, das sogenannte "nützliche Dreieck" innerhalb des Befestingungswalls nach Marokko zu integrieren und dafür die Gründung eines sahraouischen Staats im übrigen Teil zu ermöglichen. ${ }^{53}$

Hassan hält sich mit Hilfe der Geldgeber aus den USA, Saudi-Arabien und Irak ${ }^{54}$ mit Hilfe des Militärs als "ein Unterdrückungsinstrument der Monarchie "s5 und eines verzweigten Spitzelsystems ${ }^{56}$ an der Macht. Rebellionen in der Armee und Unruhen in

48 Archiv der Gegenwart vom 10. 6. 1983 (26706A); Frankfurter Rundschau (FR) 5. 9. 1983.

49 Archiv der Gegenwart 9. 7. 1983, 26795 D.

50 Vgl. die Tageszeitung 21. 10. 1983 und NZZ 23. 11. 1983.

51 Siehe Herzog in FR 5. 9. 1983, S. 11.

52 Zit. in FR 11. 7. 1983.

53 NZZ 14. 6. 1983.

54 Djaider, L'accumulation des faits, in: Afrique-Asie, No. 247 vom 31. 8. 1981, S. 22f. spricht von der Neuauflage eines Marshall-Plans. Der Irak dürfte nach Beginn des Krieges gegen Iran seine Zahlungen eingestellt haben. Nachdem auch Saudi-Arabien seine Zahlungen von jährlich etwa 2,5 Milliarden Dollar eingestellt hat, mußte Marokko als zweiter afrikanischer Staat nach dem Sudan seine Zahlungsunfähigkeit anmelden. Die Auslandsschulden belief en sich Ende 1983 auf etwa 10 Milliarden Dollar. (vgl. Handelsblatt Nr. 164, 26./ 27. 8. 1983; SZ 2. 9. 1983).

55 Tibi, a.a.O. (Anm. 35), S. 131 und ders., Zur Rolle der Armee in Marokko, in: Blätter für deutsche und internationale Politik, 17(1972)9, S. 941-960.

56 Vgl. Herzog, Des Königs Spitzel und der Wüstenkrieg, in: FR 2. 11. 1981, S. 9; zu Haftbedingungen und Folter in Marokko Afrique-Asie. No. 218 vom 21. 7. 1980, S. 8-11 und No. 247 vom 31. 8. 1981, S. 23. 
der Bevölkerung verhindert er mit Hilfe königstreuer Politiker derzeit durch eine modifizierte Neuauflage der Kampagne für "das große Maghreb".

\subsection{Mauretanien}

Mauretanien erhob erstmals 1957 geografisch und ethnisch begründete Ansprüche auf Teile der Spanischen Sahara, auf das von ihm so bezeichnete "Spanisch-Mauretanien". Anlaß für die Gebietsforderung war die Sorge u m die eigene Souveränität angesichts der in Marokko veröffentlichten Landkarte von "Groß-Marokko". Wenn schon aufgrund der aggressiven Gebietsansprüche Marokkos kein souveräner Staat Sahara möglich schien, so sollte dem mauretanischen Mutterland doch ein mauretanischer Sahara-Gürtel vorgelagert sein. Okonomische Gründe waren ebenfalls von Bedeutung. Mauretanien ist das an Bodenschätzen ärmste Land Afrikas. Die entdeckten Eisenerzlager sind sehr unwirtschaftlich. Eine Provinz Sahara würde daher die Chance auf Ol- oder Phosphatfunde erhöhen. Andererseits war der 1975 amtierende Präsident Ould Daddah eher algerienfreundlich eingestellt und in der Bevölkerung gab es unüberhörbar pro-sahraouische Stimmen. ${ }^{57}$ Den Ausschlag für eine Unterstützung Marokkos mag die Zusicherung Frankreichs gegeben haben, bei entsprechender Haltung zugunsten Marokkos das Land wirtschaftlich zu stützen. Von da an folgte Ould Daddah, der vor 1975 immer wieder das Selbstbestimmungrecht der Sahraoui betont hatte, Hassan II. eher, als daß er ihm voranging. ${ }^{58}$ Die Folge war, daß das Militär 1975 von 1500 auf 15000 Mann vergrößert wurde und die Präsenz französischer Elitesoldaten und marokkanischer Truppen begann.

Der durch die Militärausgaben drohende Staatsbankrott konnte nur durch massive Unterstützung Frankreichs, Saudi-Arabiens und der Emirate am Persischen Golf verhindert werden. ${ }^{59}$ Gegen diese wirtschaftliche Entwicklung rebellierten die Bevölkerung und Teile der Armee bereits 1976. Aber erst am 10. Juli 1978 beendete der mauretanische Generalstab die 18-jährige Herrschaft Daddahs. Mauretanien reagierte auf den darauf einseitig von der F. POLISARIO ausgerufenen Waffenstillstand zunächst nicht. Erst als die F. POLISARIO die Kampfhandlungen wieder aufnahm, führten die Friedensverhandlungen im August 1979 mit der Unterzeichnung eines Friedensvertrags zum Erfolg. ${ }^{60}$ Mauretanien konnte allerdings nicht die Ubernahme des ehemals von ihm beanspruchten und of fiziell besetzten Sahara-Gebiets durch Marokko am 14. 8. 1979 verhindern. 1979 erst zogen die letzten Truppen Marokkos aus Mauretanien ab. Ein von Marokko unterstützter Putschversuch, der ein Ende der mauretanischen Neutralitätspolitik herbeiführen sollte, schlug 1981 fehl. Die von Mauretanien daraufhin abgebro-

57 Vgl. Gretton, a.a.O. (Anm. 12), S. 37 und Franck, a.a.O. (Anm. 13), S. 706.

58 Vgl. Franck, a.a.O. (Anm. 13), S. 706 und jeune afrique, No. 790 vom 27. 2. 1976, S. 26 f.: Mokhtar Ould Daddah, Sahara-La genèse d'un affrontement.

59 Vgl. Meyer, Eine Chance für den Frieden?, in: 3. Welt Magazin, (1979), S. 18-20.

60 W. Sahara, What next?, in: Africa (London), (1979)97, S. 18f.; FR 7. 8., 13. 8. und 14. 8. 1979. 
chenen diplomatischen Beziehungen zu Marokko wurden inzwischen durch Vermittlung von Kronprinz Fahd von Saudi-Arabien im Anschluß an die 18. OAU-Gipfelkonferenz wieder aufgenommen; ein bilateraler Vertrag verpflichtet die Länder zur gegenseitigen Nichteinmischung. Obwohl Marokko auf mauretanisches Gebiet vordrang und dort Bombenangriffe gegen zivile Objekte flog, hält Mauretanien derzeit an seiner Neutralitätspolitik fest. Die Beziehungen Mauretaniens zur F. POLISARIO sind freundlich, eine diplomatische Anerkennung der DAR Sahara erfolgte allerdings bisher nicht.

\subsection{Algerien}

Algerien konnte jahrelange Grenzauseinandersetzungen mit Marokko erst 1972 durch die Unterzeichnung eines Grenzvertrags während der 9. OAU-Gipfelkonferenz beenden. Darin verzichtete Marokko auf Gebietsansprüche gegenüber Algerien, bekam aber von diesem die Beteiligung an der Erschließung von Bodenschätzen im umstrittenen Gebiet zugesprochen. $1976 \mathrm{kam}$ es noch einmal zu militärischen Auseinandersetzungen, als algerische Soldaten einen Verpflegungs- und Medikamentenkonvoi zu einem sahraouischen Flüchtlingslager begleiteten und dabei auf algerischem Gebiet von marokkanischen Truppen angegriffen wurden. Algerien regagierte mit dem vorübergehenden Abbruch der diplomatischen Beziehungen.

Sowohl die Politik Boumedienne's als auch die Chadli Ben Jedid's war immer darauf gerichtet, am Prinzip des Selbstbestimmungsrechts entkolonialisierter Länder festzuhalten. ${ }^{61}$

\subsection{Frankreich}

Im Westsahara-Krieg hat Frankreich Partei für Marokko ergriffen und es schon vor Abzug der Spanier mit Waffen und Lebensmitteln versorgt. U. a. lieferte Frankreich Mirage-Flugzeuge, Lastwagen und andere militärische Güter.$^{62}$ Frankreich griff auch selbst militärisch in den Krieg ein, indem es Aufklärungsflüge über der Westsahara flog und hohe Offiziere als Berater nach Marokko schickte. Auf der Seite Mauretaniens flog die französische Luftwaffe 1977 mit im Senegal stationierten Kampfflugzeugen Luftangriffe gegen F. POLISARIO-Einheiten. ${ }^{63}$ Auf diplomatischer Ebene hat Frankreichs Regierung sich geweigert, die F. POLISARIO als Gesprächspartner und das Selbstbe-

61 Die Darstellung Algeriens als expansionistischer Aggressor in Nordafrika, der unter Beruf ung auf das Selbstbestimmungsrecht der Völker an die eigene Ausdehnung denke und diese auch kriegerisch durchzusetzen bereit sei, hält einer Uberprüfung nicht stand. So aber Eikenberg, Fragen des Maghreb und der Entkolonisierung der Spanischen Sahara, in: Krämer (Hg.), Afrika Wirtschaft (1976/77), S. 35-47(46f.).

62 Vgl. Junqua, France-Algerie: De l'idylle à la crise, I-IV, in: Le monde 27.-30. 1. 1976.

63 Vgl. Hodges, Western Sahara, The escalating confrontation, in: Africa Report, 23(1978)2, S. 4-9 und ders., French entanglement grows in Polisario war, in: New African (1978), S. 16-18. 
stimmungsrecht der Sahraoui anzuerkennen. In internationalen Organisationen hat es zugunsten Marokkos gestimmt. ${ }^{64}$

Die neue Regierung Mitterrand hat durch ihren sozialistischen Außenminister Claude Cheysson erklärt, daß seine Regierung an den guten Beziehungen zu Marokko festhalten will. Er hat bei seinem Besuch in Algerien im August 1981 aber auch seine Genugtuung über die OAU-Resolution zum Sahara-Referendum zum Ausdruck gebracht und gewarnt, daß ein Rückzug Marokkos aus seiner Verpflichtung die Beziehung mit Frankreich schwer belasten würde. ${ }^{65}$

Nach den Unruhen und Massenverhaftungen 1981 in Marokko hat das französische Außenministerium eine scharfe Erklärung zu den Verhaftungen übermittelt und auf Marokkos Verpflichtung zur Durchführung des Referendums hingewiesen. ${ }^{66}$

Die Anerkennung der F. POLISARIO durch die sozialistische Partei Frankreichs hat allerdings nicht die Anerkennung der Demokratischen Arabischen Republik Sahara durch die sozialistische Regierung zur Folge gehabt.

Das wirtschaftliche und militärische Interesse an Marokko veranlaßt Frankreich zu einer weitgehend neutralen Haltung im Westsahara-Krieg. Von Frankreich gehen derzeit keine Initiativen aus, um in der Sahara-Region Frieden und Stabilität schnellstmöglich herbeizuführen.

\subsection{USA}

Die USA unterstützen Marokko, das als militärischer Verbündeter der NATO gilt, seit 1960 durch Waffenlieferungen und Wirtschaftshilfe. Marokko ermöglicht den USA die Unterhaltung zahlreicher Militärstützpunkte ${ }^{67}$ und bildete in der Vergangenheit auch militärische Eingreifgruppen in Afrika im Interesse des Westens, beispielsweise in Zaire während der Shaba-Rebellion 1977/78. ${ }^{68} 1979$ hatte der ehemalige US-Außenminister Vance nach einem Empfang der Außenminister Marokkos und Algeriens erklärt, die USA würden sich in dem Sahara-Konflikt neutral verhalten. Vance berücksichtigte dabei wirtschaftliche Interessen gegenüber Algerien, von dem die USA einen großen Teil ihres Olbedarfs importieren. Dennoch beabsichtigten die USA noch zu Zeiten der Carter-Administration, ihren 19 Jahre alten Vertrag über die Lieferung von Rüstungsgütern an Marokko nach dessen Einzug in den von Mauretanien verlassenen Teil der Westsahara zu ändern. Durften die US-Waffen von Marokko zuvor nur im Innern zur Abwehr gegen Aggressoren eingesetzt werden - woran sich Marokko nicht gehalten

64 S. den Bericht im 'Zeit-Magazin` vom 13. 4. 1978 und Europäische Zeitung, (1981)4, S. 10; EP Dok. 1-532/ 80.

65 Le monde 11. 8. 1981.

66 Le monde $13 . / 14$. und 16. 9. 1979.

$67 \mathrm{Vgl}$. Hultman, The struggle for Western Sahara, in: ISSUE: A Quarterly Journal of Africanist Opinion, 7(1977)1, S. 27-32; FR 24. 10. 1979; NZZ 25. und 31. 10. 1979; The Guardian 7. 3. 1979.

$68 \mathrm{Vgl}$. Europa-Archiv, Zeittafel 1978, Z $128 \mathrm{ff}$. 
hatte -, sollten nun auch legal Cobra-Helikopter und Kampfflugzeuge zum Einsatz in der Westsahara geliefert werden können. Bediente sich die USA zur Lieferung von Militärmaterial bis dahin der Unterstützung von Drittländern, wie etwa Ägyptens, ${ }^{69}$ so wurden nun auch moderne amerikanische Kampfflugzeuge und Flugzeuge für die Luftunterstützung von Bodentruppen ${ }^{70}$ direkt an Marokko geliefert. Der Umfang der Lieferungen entsprach aber immer noch nicht den weitergehenden Wünschen Hassans. Mit dem Amtsantritt Ronald Reagans wurden die Militärhilfe und die wirtschaftspolitischen Beziehungen mit dem Ziel der kompromißlosen "Funktionalisierung von Wirtschafts- und Militärhilfe für außen- und sicherheitspolitische Ziele und Zwecke " ${ }^{71}$ der USA ausgebaut. Reagan erklärte die Entspannungspolitik endgültig für gescheitert und stockte die Rüstungspläne auf. Gleich nach seinem Amtsantritt veranlaßte der US-Präsident im Januar 1981 die Lieferung von 108 Panzerfahrzeugen, 120 Jagdbombern und 6 Aufklärungsflugzeugen an Marokko. Gleichzeitig erklärte er diese Unterstützung noch für ungenügend.

Am 27. Mai 1982 kam es zur Unterzeichnung des amerikanisch-marokkanischen Militärabkommens, wonach die Vereinigten Staaten für die Dauer von zunächst 6 Jahren Nutzungsrechte auf 2 marokkanischen Luftwaffenbasen erhalten, die mit amerikanischer Investitionshilfe in Höhe von 20 Millionen Dollar modernisiert werden sollen. Seit dieser Zeit sind US-Militärberater an der Leitung der Operationen der marokkanischen Streitkräfte beteiligt, US-Satelliten verfolgen die Bewegungen der F. POLISARIO in der Sahara, marokkanische Offiziere und Soldaten werden von US-Beratern ausgebildet und außerdem fanden in den letzten zwei Jahren gemeinsame amerikanischmarokkanische Truppenübungen einschließlich der Beteiligung der 6. amerikanischen Flotte statt. Zur Sicherung des marokkanischen Verteidigungwalls rüsteten die USA Marokko mit umfangreichem elektronischen Material aus. Zuletzt setzte Marokko auch völkerrechtlich geächtete und von den USA gelieferte Splitterbomben im Kampf gegen die F. POLISARIO ein. Von besonderer Bedeutung für die globale US-Militärstrategie ist Marokko als Stützpunkt der "Schnellen Eingreiftruppe" (Rapid Deployment Force), mit der beispielsweise die Olfördergebiete am Persischen Golf unter Kontrolle gebracht werden sollen, wenn anders die Olversorgung nicht mehr möglich wäre. ${ }^{72}$

Die Vereinigten Staaten haben die Gewährung ihrer Militärhilfe an Marokko allerdings davon abhängig gemacht, daß dem US-Kapital Investitionsmöglichkeiten in Marokko eröffnet werden. Ein entsprechendes marokkanisches Gesetz hat diese Möglichkeit inzwi-

69 FR 3. 9. 1979; NZZ 11. 9. 1979; vgl. auch Hottinger, a.a.O. (Anm. 36), S. 657, 660. Die Waffenlief erungen aus Ägypten erfolgten beispielsweise auch noch im September 1979, obwohl Marokko die diplomatischen Beziehungen wegen der ägyptischen Israel-Politik am 26. April 1979 abgebrochen hatte.

70 Le monde 27. 3. 1981; FR 28. 10. 1981.

71 Nuscheler, Reaganism und Dritte Welt - Neue Rhetorik oder entwicklungspolitische Wende;, in: Dt. Ubersee-Institut (Hg.), Jahrbuch Dritte Welt 1, München 1983, S. 73-86(75).

72 Vgl. Kunig, a.a.O. (Anm. 25), S. 235,353; Nuscheler a.a.O. (Anm. 71), S. 77, 83 und Tzschaschel, Wege und Wirkungen der Außenpolitik Marokkos, in: Europa-Archiv, 15(1983), S. 453-460(457). 
schen geschaffen. Die Verfolgung der Wirtschaftsinteressen in Marokko entspricht der allgemeinen Reagan-Politik in der Dritten Welt, überall Handels- und Investitionsbarrieren aus dem Weg zu räumen. Auch die Waffen sollten nicht mehr verschenkt, sondern mit Hilfe zinsvergünstigter und öffentlich garantierter Kredite verkauft werden. Diese mit wirtschaftspolitischen Interessen unmittelbar verknüpften militärischen Hilfen haben die wirtschaftliche Situation für Marokko weiter verschlechtert. Die USA haben Marokko im Sommer letzten Jahres ultimativ die Einstellung aller Wirtschafts- und Militärhilfe für den Fall angekündigt, daß Marokko seine Zinsschuld über 10 Millionen Dollar Rüstungskredite nicht bezahlt.

Auch in den USA blieb die Marokko-Politik der USA nicht ohne Kritik. ${ }^{73}$ Verschiedene politische Organisationen haben die Erhöhung der Militärhilfe an Marokko kritisiert, das Selbstbestimmungsrecht des sahraouischen Volkes unterstützt und daran erinnert, daß sich die USA offiziell im Sahara-Krieg nach wie vor für neutral erkläre. Der Unterausschuß des amerikanischen Kongresses hat jüngst eine radikale Kürzung der Militärhilfe an Marokko gefordert.

\subsection{Andere Staaten}

Außer auf die Haltungen der Hauptakteure im Konflikt soll kurz auf die der Sowjetunion und der Bundesrepublik eingegangen werden.

Die Sowjetunion hatte 1975 in den UN die algerische und nicht die marokkanische Resolution unterstützt, die den Vertrag zwischen Spanien und Marokko/Mauretanien über die Aufteilung der Sahara von 1975 nicht anerkennt. Noch 1976 aber verschwieg sie die Existenz der F. POLISARIO und verbreitete, es gäbe in der Sahara zahlreiche, über Kampfmethoden und politische Zukunft untereinander zerstrittene Befreiungsbewegungen. ${ }^{74}$ Während etwa Kuba und Nordkorea die Republik Sahara diplomatisch anerkannt haben, hat sich die UdSSR gegenüber Marokko in einem Vertrag über die Lieferung von Eisenerzen zur Neutralität im Sahara-Konflikt verpflichtet. ${ }^{75}$ Die Sowjetunion ist an ungetrübten politischen und wirtschaftlichen Beziehungen zu Marokko interessiert. ${ }^{76}$

Die Bundesrepublik Deutschland pflegt mit Marokko ein freundschaftliches Verhältnis. Sie hat an Marokko zwischen 1968 und 1982 Ausrüstungshilfe in Höhe von 37 Millionen DM gezahlt. In einem Dreijahresprogramm 1982-1984 ist vorgesehen, 8 Millionen DM zur Verbesserung der Bedingungen auf dem Versorgungssektor an Marokko zu zahlen. Verbessert werden sollen damit die Versorgungsmöglichkeiten der Streitkräfte

73 Vgl. nur 'Arms for Morocco?, Report of a Study Mission to the Committee on Foreign Affairs, U.S. House of Representatives, Januar 1980 (55-483-0).

74 Gurenko, Western Sahara, in: International Affairs, (1976)2, S. $147 f$.

75 Vgl. jeune af rique 26. 7. 1978 und Le monde 8. 7. 1981. Der marokkanische KP-Abgeordnete bestätigt die marokkofreundliche Haltung der UdSSR in jeune af rique, 2. 4. 1980, S. $28 \mathrm{f}$.

$76 \mathrm{Vgl}$. Roosens, La question du Sahara occidental, in: Studia Diplomatica, Brüssel, 32(1979)5, S. 513-535(518); Vellas, La diplomatie marocaine dans l'aff aire du Sahara occidental, in: Politique étrangère, 43(1978)4, S. 417-428; Clausen a.a.O. (Anm. 8), S. 94f. und Tzschaschel, a.a.O. (Anm. 72), S. 457. 
durch Feldküchen, Bäckereien und Kühlwagen. ${ }^{77}$ Bundesdeutsche Konzerne werden beschuldigt, Waffen an Marokko für den Einsatz in der Sahara geliefert zu haben. Die Staatsanwaltschaft Düsseldorf ermittelt nach einer Durchsuchung beim Rüstungskonzern Rheinmetall wegen Verstoßes gegen das Kriegswaffenkontrollgesetz aufgrund der Lieferungen von Maschinenkanonen an Marokko. ${ }^{78}$

Die Demokratische Arabische Republik Sahara wird derzeit von 56 Staaten, darunter 26 afrikanischen, diplomatisch anerkannt.

\section{Die Behandlung des Konflikts durch die Vereinten Nationen}

Die Vereinten Nationen nahmen sich der Westsahara-Frage erstmals 1963 an, als Spanien das Gebiet nicht mehr als Kolonie, sondern als Provinz des Mutterlandes betrachtete. $^{79} 1965$ verabschiedete die Generalversammlung eine erste Resolution, mit der Spanien aufgefordert wurde, in der Spanischen Sahara und in der Enklave Ifni die "notwendigen Maßnahmen für die Befreiung dieser Gebiete vom Kolonialismus" zu treffen. ${ }^{80}$ Die Generalversammlung nahm dabei Bezug auf die Resolution 1514(XV) vom 14. 12. 1960 mit der "Erklärung über die Gewährung der Unabhängigkeit an koloniale Länder und Völker“. Danach haben alle Völker das Recht auf Selbstbestimmung, bestimmen frei ihren politischen Status und verfolgen frei ihre wirtschaftliche, gesellschaftliche und kulturelle Entwicklung. In den folgenden Jahren verabschiedete die Generalversammlung weitere Resolutionen, in denen Spanien zur Vorbereitung und dann dringlicher zur Durchführung eines Referendums aufgefordert wurde. Die grundsätzliche Gewährung und die Form der Selbstbestimmung war auch in zahlreichen Kommuniqués unbestritten. ${ }^{81}$

Der erstmals in der Resolution 2229(XXI) vom Dezember 1966 gebrauchte Wortlaut, wonach die Durchführung des Referendums durch Spanien "in Abstimmung mit der Bevölkerung, der marokkanischen und der mauretanischen Regierung sowie allen anderen interessierten Parteien" geschehen solle, ist eine völkerrechtliche Besonderheit. In allen anderen Dekolonialisierungs-Resolutionen war nur die Rede von dem zu dekolonialisierenden Gebiet sowie der Kolonialmacht. Hier wurden auch weitere, an der Dekolonialisierung interessierte, teilweise Gebietsansprüche geltend machende dritte

77 Wehrdienst-Informationsbrief für die Rüstungswirtschaft, Nr. 682/78 und Nr. 848/82; Das Statistische Bundesamt hat in ihrem "Länderkurzbericht Marokko 1982", S. 10, die of fizielle marokkanische Version übernommen, wonach "der nördliche Teil von ehemals Spanisch-Sahara . . . nach Freigabe durch Spanien an Marokko gefallen ist".

78 Weser-Kurier 25. 11. 1982; Der SPIEGEL Nr. 35/1983, S. 89f.

79 Vgl. Shaw, The Western Sahara Case, in: The British Yearbook of International Law, 49(1978), S. 119-154(121) und Franck, a.a.O. (Anm. 13), S. 698.

80 GA Res. 2072(XX) vom 16. 12. 1965.

81 Vgl. Hinz, Le droit à l'autodetermination du Sahara Occidental. Le chemin difficile du peuple Sahraoui, Bonn 1978, S. 21 ff. und passim. 
Parteien mit in die Resolution aufgenommen. Sureda erklärt diesen Umstand damit, daß nicht deutlich sei, ob die Spanische Sahara nicht ähnliche Bedingungen aufweise wie die Enklave Ifni. ${ }^{82}$ Suredas Erklärung für diesen ungewöhnlichen, auf mauretanischen Druck zustande gekommenen Passus erscheint wenig überzeugend. Richtig ist zwar, daß bei Enklaven die Rechtsregeln über das Selbstbestimmungsrecht nicht anzuwenden sind, die Enklaven vielmehr an das sie umgebende Land zurückgegeben werden. Nur ist die Westsahara ohne Zweifel keine Enklave. Auch die Generalversammlung der Vereinten Nationen hat zu keinem Zeitpunkt die Spanische Sahara als eine Enklave betrachtet; nur Ifni, das alsbald nach Verabschiedung der Resolution von Spanien an Marokko zurückgegeben wurde, ist ausdrücklich als Enklave bezeichnet worden. Die ehemalige Spanische Sahara ist zudem von mehreren Anliegerstaaten umgeben, so daß auch geographisch nicht von einer Enklave gesprochen werden kann. Schließlich ist auch das Argument Suredas, der Umstand der geringen Bevölkerungszahl der Sahara habe den Wortlaut der UN-Resolution beeinflußt, nicht begründet, haben doch Staaten mit wesentlich geringerer Bevölkerungszahl, wie beispielsweise in Afrika die Seychellen, ihre Souveränität erlangt.

Als Erklärungshypothese für die von gleichgelagerten Fällen abweichende Formulierung der Resolution ist eine andere Úberlegung geeigneter. Im Unterschied zu kolonialen Konflikten (etwa Portugal-Angola, Frankreich-Algerien) bestand der Konflikt nicht mehr im "Nord-Süd-Gegensatz", also dem Verhältnis der Westsahara zur Kolonialmacht Spanien. Vielmehr wurde im vorliegenden Fall das Recht auf Selbstbestimmung von afrikanischen Nachbarn, die früher selbst Kolonien waren, bestritten und von diesen eigene Gebietsansprüche erhoben. Hinzu kommt noch ein anderer Umstand. Die unüberhörbare Darstellung des sahraouischen Rechts und wirkungsvolle Versuche, diesem zur Durchsetzung zu verhelfen, setzten erst mit der Formierung der F. POLISARIO 1972 ein. Bis dahin mag die Bevölkerung der Westsahara auch von den Vereinten Nationen nicht in der notwendigen Weise wahrgenommen und damit nicht in den Resolutionen entsprechend berücksichtigt worden sein. Dafür spricht auch, daß es nach der Durchführung des Ersten Allgemeinen Volkskongresses in der Sahara im Mai 1973 und nach dem Zweiten Kongreß im August 1974 zu einer politischen Wende bei den an der Sahara interessierten Staaten kam. Beispielsweise sah Spanien seine Hoffnungen auf fortdauernde Integration der Sahara in das Mutterland schwinden und Marokko seine wirtschaftlichen Interessen an der Ausbeutung der Bodenschätze.

Die Vereinten Nationen haben demnach zwar seit 1963 immer wieder die Dekolonisierung von Spanien gefordert und das Recht auf Selbstbestimmung der Sahraouis betont ${ }^{83}$ aber gleichzeitig sind sie von der üblichen Praxis abgewichen, indem sie in der

82 Sureda, The evolution of self-determination, Leiden 1973.

83 Vgl. Hinz, a.a.O. (Anm. 81), S. 21 ff.; zuletzt verabschiedete die 38. Vollversammlung der UN am 17. November 1983 einstimmig die folgende Resolution: 
Formulierung der Resolution zum Ausdruck brachten, daß andere interessierte Parteien zu beachten seien. Die UN lassen im Westsahara-Konflikt zumindest in der Abfassung des Resolutionswortlauts die in anderen Entkolonialisierungsfällen geübte Konsequenz vermissen.

Auf die schon angesprochene politische Wende ab 1973 ist es zurückzuführen, daß es in den UN in der Behandlung der Westsahara 1974 plötzlich "juristische Schwierigkeiten" gab, die eine sich an frühere Fassungen anlehnende Resolution nicht mehr zuließ. Um diese Schwierigkeiten zu klären, nahmen die Vereinten Nationen am 13. Dezember 1974 die Resolution 3292(XXIX) ${ }^{84}$ auf Vorschlag Marokkos an, vom Internationalen Gerichtshof in Den Haag ein Rechtsgutachten zur Westsahara-Frage einzuholen ${ }^{85}$ und eine

Die Vollversammlung:

1. nimmt den Bericht des Durchführungskomitees der OAU zur Westsahara-Frage zur Kenntnis;

2. fordert die Konfliktparteien, das Königreich Marokko und die Frente Polisario, auf, direkte Verhandlungen aufzunehmen, um einen Waffenstillstand zu erlangen, der die notwendigen Bedingungen für ein friedliches und gerechtes Referendum im Hinblick auf die Selbstbestimmung des Volkes der Westsahara schafft. Das Referendum soll ohne jegliche militärische oder administrative Einschränkungen unter der Schirmherrschaft der OAU und der UNO stattfinden. Die Vollversammlung bittet das Durchführungskomitee, die Einhaltung des Waffenstillstandes zu überwachen;

3. ersucht das Durchführungskomitee sich so schnell wie möglich und in Abstimmung mit den Konfliktparteien zu treffen, um die Modalitäten und andere wichtige Details im Hinblick auf die Durchführung des Waffenstillstandes und der Organisation des Referendums im Dezember 1983 festzulegen;

4. bittet die Vereinten Nationen, Sicherheitskräfte in die Westsahara zu entsenden, um den Frieden und die Sicherheit während der Organisation und der Abwicklung des Referendums zu garantieren;

5. beauftragt das Durchführungskomitee in Zusammenarbeit mit den Vereinten Nationen, alle notwendigen Maßnahmen zu ergreifen, um die korrekte Durchführung dieser Resolution sicherzustellen;

6. bittet das Durchführungskomitee, der 20. OAU-Gipfelkonferenz der Staats- und Regierungschefs die Ergebnisse des Referendums mitzuteilen, um der 20. Gipfelkonferenz eine abschließende Entscheidung über die Westsahara-Frage zu ermöglichen;

7. beschließt, sich weiterhin mit der Westsahara-Frage zu befassen;

8. bittet das Durchführungskomitee im Rahmen seines Mandats, die Verhandlungsprotokolle der 18. und 19. ordentlichen Sitzungen der OAU über die Westsahara zu berücksichtigen und ersucht den Generalsekretär der OAU, dem Komitee alle entsprechenden Verhandlungsprotokolle zur Verfügung zu stellen;

9. begrüßt die konstruktive Haltung der sahrauischen Fühirung, die durch ihren freiwilligen und vorübergehenden Rückzug das 19. Gipfeltreffen ermöglicht hat;

10. bittet den UN-Generalsekretär, die notwendigen Maßnahmen zu ergreifen um eine effektive Teilnahme der Vereinten Nationen bei der Organisation und der Durchführung des Referendums sicherzustellen und darüber der Vollversammlung und dem Sicherheitsrat Bericht zu erstatten, einschließlich der Maßnahmen, die eine Entscheidung des Sicherheitsrates erfordern;

11. bittet eindringlich den UN-Generalsekretär, eng mit dem OAU-Generalsekretär zusammenzuarbeiten, um die Umsetzung der entsprechenden Beschlüsse sowohl der OAU als auch dieser Resolution sicherzustellen;

12. bittet das Komitee für Dekolonialisierung weiterhin die Situation in der Westsahara als vordringliche Frage zu behandeln und der Vollversammlung anläßlich ihrer 39. Sitzung darüber Bericht zu erstatten.

84 Abgedruckt in: Vereinte Nationen, 23(1975)6, S. 189.

85 Western Sahara, Order of 3 January 1975, I.C.J. Reports 1975; Kurzfassung des Gutachtens mit deutschen Leitsätzen in: Archiv des Völkerrechts, (1978-1980), S. 137-142; Vgl. auch Magiera, Die Rechtsprechung des Internationalen Gerichtshofes im Jahre 1975, in: German Yearbook of International Law, 19(1976), S. 443-454; Flory, L'avis de la Cour Internationale de Justice sur le Sahara Occidental, in: Annuaire Français de Droit International, 21(1975), S. 253-277; Ndiaye, Avis de la C.I.J. sur le Sahara occidental, 16 octobre 1975, in: Revue Sénégalaise de Droit, 10(1976)19, S. 31-53; Chemillier-Gendreau, La question du Sahara occidental, in Annuaire du tiers monde, Paris, (1975/76)2, S. 270-280; Vallée, L'affaire du Sahara occidental 
Mission in das Territorium zu entsenden, die der nächsten Generalversammlung einen Situationsbericht erstellen sollte.

Der Gerichtshof in Den Haag mußte zunächst prüfen, ob er überhaupt die Kompetenz hatte, zu den ihm vorgelegten Fragen ein Gutachten zu erstellen.

Die Fragen lauteten:

"1. War die West-Sahara (Rio de Oro und Sakiet El Hamra) zur Zeit der Kolonisierung durch Spanien res nullius`?

Wenn die Antwort auf diese erste Frage verneint wird:

2. Welche rechtlichen Bande bestehen zwischen dem Gebiet und dem Königreich Marokko einerseits und Mauretanien andererseits?"

Wie immer man im Gutachten oder schon in der Fragestellung das Verhältnis zwischen rechtlichem und politischem Inhalt sieht, so war doch von Anfang an sichtbar, daß ein Gutachten keine praktische Bedeutung haben würde. Würde das Gericht feststellen, daß die Sahara kein terra nullius' (und keine Enklave) ist und keine rechtlichen Bindungen zu den Nachbarn vorhanden sind, so bleiben immer noch mehrere Möglichkeiten, zu welcher Zukunft sich die Bevölkerung in Wahrnehmung des Selbstbestimmungsrechts entscheiden würde: zu einem unabhängigen Staat, zu einer Assoziation mit einem anderen unabhängigen Staat oder zu einer Integration in einen anderen unabhängigen Staat. Uber die Zukunft aber hat das Gericht seinem Auftrag entsprechend keine

devant la Cour International de Justice, in: Maghreb-Machrek, (1976)71, S. 47-55; Condorelli, Le droit international face a l'autodetermination du Sahara occidental, in: La Comunità Internazionale, 33(1978)3, S. 396-405; Barbier, L'avis consultatif de la Cour de la Haye sur le Sahara, in: Revue Juridique et Politique, Indépendence et Cooperation, 30(1976)1, S. 67-103; Blaydes, International Court of Justice does not find legal ties' of such a nature to affect self-determination in the decolonization process of Western Sahara, in: Texas International Law Journal, 11(1976)2, S. 354-386; McWhinney, Judicial Opinion Writing in the World Court and the Western Sahara Advisory Opinion, in: Zeitschrift für ausländisches öf entliches Recht und Völkerrecht, (1977)37, S. 1-42; Janis, The International Court of Justice: Advisory Opinion on the Western Sahara, in: Harvard International Law Journal, 17(1976)73, S. 609-621; Levy, Advisory opinion on the western Sahara, in: Brooklyn Journal of International Law, 2(1976)2, S. 289-307; van Dongen, Het Hof en de Spaanse Sahara, Vonnis of advies?, in: Nederlands Juristenblad, 50(1975)44/45, S. 1439-1447; Carillo Salcedo, Libre determinacion de los pueblos e integridad territorial de los estados en el dictamen del Tribunal Internacional de Justicia sobre el Sahara Occidental, in: Revista española de Derecho Internacional, 29(1976)1, S. 33-49; Bedjaoui, Terra nullius, 'droits' historiques et autodétermination, Den Haag 1975; Chappez, L'avis consultatif de la Cour internationale de justice du 16 octobre 1975 dans l'affaire du Sahara occidental, in: Revue générale de droit international public, 80(1976)4, S. 1132-1187; Bennouna, L'affaire du Sahara occidental devant la Cour internationale de justice, in: Revue juridique, politique et économique du Maroc, (1976)1, S. 81-106; Isoart, Reflexions sur les liens juridiques unissant le Maroc et le Sahara occidental, ebenda, (1978)4, S. 11-47; Mathy, Un juge ad hoc en procédure consultative devant la Cour internationale de justice, in: Revue belge de droit international, 12(1976)2, S. 528-554; Pinto, Affaire du Sahara occidental, in: Journal du droit international, 102(1975)4, S. 873-882; Prévost, Observations sur l'avis consultatif de la Cour international de justice relatif au Sahara occidental, ebenda, 103(1976)4, S. 831-862; Riedel, Confrontation in Western Sahara in the light of the advisory opinion of the international Court of justice of 16 th October 1975. A critical appraisal, in: German Yearbook of International Law, 19(1976), S. 405-442; Parzymies, Spór wokól Sahary Zacodniej, in: Sprawy Miedzynarodowe (Warschau), 29(1976)4, S. 79-94. 
Auskunft gegeben. ${ }^{86}$ So bleibt schon vor der Fragestellung durch die UN festzustellen, daß das Gutachten eher der besseren Information der Vereinten Nationen über historische und politische Fragen dienen würde. ${ }^{87}$ Dementsprechend konnte der Gutachtenauftrag 1974 nur eine für Marokko günstige zeitliche Verschiebung der Behandlung und der Entscheidung des Problems durch die UN bedeuten.

Am 16. Oktober 1975 gab der IGH sein Gutachten bekannt. Das Gericht ist danach der Ansicht, daß zur Zeit der Kolonisierung kein 'terra nullius` vorhanden gewesen ist. Sich daher der zweiten Frage zuwendend, erklärte es nach einer sehr ausführlichen Auswertung des ihm unterbreiteten Materials weiter, daß zwischen der Westsahara und Marokko sowie Mauretanien zwar gewisse rechtliche Beziehungen bestanden hätten. Diese seien aber nicht von solcher Art, daß sie eine Souveränitätsausübung von der einen oder anderen Seite aus implizierten. Aus diesem Grunde sah der Gerichtshof keinen Anlaß dafür, daß die UN-Resolutionen über die Entkolonisierung nicht angewandt werden könnten. Die Entscheidung entsprach damit der zuvor von Spanien, Algerien und der F. POLISARIO vertretenen Auffassung.

Der Wert einer IGH-Entscheidung bei Streitigkeiten in internationalen Angelegenheiten entfaltet sich erst bei freiwilliger Unterwerfung unter einen Schiedsspruch und bei Gutachten in der Verwendbarkeit im diplomatischen Tauziehen. ${ }^{88}$ Das zeigt auch das Beispiel des Sahara-Gutachtens. Obwohl es der (Rechts-)Auffassung von Hassan II. widersprach, interpretierte der Monarch es in seinem Sinne und stützte auf diese Interpretation seine weitere Propaganda. Um der Ausübung des Selbstbestimmungsrechts der Sahraoui vollendete Tatsachen entgegensetzen zu können, rief er noch am 16. Oktober $1975 \mathrm{zu}$ einem von ihm so bezeichneten 'Friedensmarsch ("Grüner Marsch") in die Westsahara auf.

Es ist im Zusammenhang mit dem Gutachten des IGH noch auf dessen Interpretation des 'terra nullius' einzugehen, da sie auch für die Frage von Bedeutung ist, ob in der Westsahara ein das Selbstbestimmungsrecht wahrnehmendes 'Staatsvolk' vorhanden ist. Die Richter nahmen radikal von der insbesondere von westlichen Juristen gebildeten Doktrin Abstand, wonach 'terra nullius' ein Gebiet sei, dessen Zivilisation nach dem Verständnis des herrschenden europäischen Rechts rückständig sei. Der IGH ließ es dahingestellt sein, welche unterschiedlichen Auffassungen es unter Juristen auch immer gegeben haben mag. Die Staatenpraxis zeige, daß von Stämmen oder einem Volk mit sozialer und kultureller Organisation bewohnte Gebiete nicht als iterra nullius betrachtet würden. Die Differenzierung scivilised/uncivilised' (nach traditionellem europäischem Verständnis) wurde also vom Gericht ersetzt durch die Frage, ob auf dem

86 Vgl. die ergänzende Stellungnahme von Richter Singh, I.C.J. Reports, S. 70-82(72) und Stewart, Much ado about nothing, in: West Africa, (1975)3045, S. 129f. und 3046, S. 1333.

87 Auf ein anderes Problem übertragen wurde das Sahara-Gutachten von Sutton, The Treaty of Waitangi Today, in: Victoriy University of Wellington Law Review, 11(1981)1, S. 17-40(19f.).

88 Dazu Merills, The justiciability of International Disputes, in: The Canadian Bar Review, 4(1969), S. 241-269(268). 
fraglichen Territorium a social and political organization nisierung einer Gesellschaftsform, die zwar nicht europäischen Maßstäben entspricht, zur Kolonialzeit gleichwohl demokratischer als die der Kolonialmacht Spaniens war, hat jedenfalls vor der Kolonisierung auf dem fraglichen Gebiet stattgefunden. ${ }^{90}$

Zwei Tage vor Veröffentlichung des IGH-Gutachtens hatte die UN-Sonderkommission, die von Mai bis Juni 1975 durch Spanien, Marokko, Algerien, Mauretanien und die Spanische Sahara gereist war, ihren Bericht vorgelegt. Darin heißt es:

"Innerhalb des Landes konnte die Untersuchungsgruppe feststellen, daß die Bevölkerung oder zumindest nahezu alle Personen, mit denen sie zusammentraf, sich ganz eindeutig für die Unabhängigkeit und gegen die Gebietsansprüche Marokkos und Mauretaniens aussprach. Sie brachten den Wunsch zum Ausdruck, daß die U.N., die OAU und die Arabische Liga ihnen Unterstützung zusagen sollen, die Unabhängigkeit zu erringen und zu bewahren. Die Frente POLISARIO, die vor der Ankunft der Untersuchungskommission als eine Art Sekte betrachtet wurde, erwies sich als die beherrschende politische Kraft in diesem Land. Die Delegation konnte allenorts Gruppierungen beobachten, die die Frente unterstützten. ${ }^{91}$

Der gegen die eindeutige Haltung der UN durchgeführte 'Grüne Marsch ließ den Sicherheitsrat zusammentreten, der den Marsch "bedauerte ${ }^{92}$ und den Generalsekretär Waldheim beauftragte, mit den Parteien zu verhandeln. Die nächsten Jahre befaßten sich die UN in verschiedenen Gremien immer wieder mit der Westsahara-Frage, bedauerten dabei die Verschärfung der Situation, baten Marokko um Teilnahme an der Friedensdynamik und empfahlen die Teilhabe der F. POLISARIO an einer gerechten Lösung. Die kontinuierliche Verabschiedung von Resolutionen verschiedener UN-Organe, Gutachten und Kommissionsberichten über 10 Jahre hinweg haben Spanien nicht veranlassen können, die Westsahara in die Unabhängigkeit zu entlassen bzw. der Bevölkerung das Recht auf Selbstbestimmung zu gewähren. Die Vorschläge der UN, unter der Kontrolle von UN-Gremien ein halbes Jahr die Kämpfe zu beenden, bevor dann unter UN-Aufsicht das Referendum durchgeführt würde, verhallten ungehört.

Die Erfolglosigkeit der UN sind ein deutlicher Hinweis auf ihre Unfähigkeit, Konflikte zu regeln, wenn bereits einer der Staaten nicht bereit ist, sich den Regeln der Staatengemeinschaft $\mathrm{zu}$ unterwerfen..$^{93}$

89 I.C.J. Reports, S. 39.

90 Vor Gericht faß3te der algerische Gesandte Bedjaoui die Entwicklung der Lehre des 'terra nullius' polemisch und in einer bildhaften Diskreditierung der europäischen Völkerrechtslehre zusammen: "- Dans l'Antiquité romaine, est nullius tout territoire qui n'est pas romain. - A l'époque des grandes découvertes des XVe et

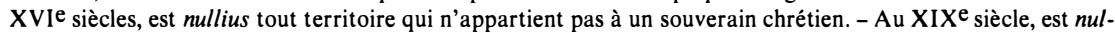
lius tout territoire qui n'appartient pas à un Etat civilisé.", in: Bedjaoui, a.a.O. (Anm. 85), S. 12 f.

91 A.a.O. (Anm. 15).

92 Entschließungen des Sicherheitsrats vom 2. 11. 1975, 380 (1975), auch in: Vereinte Nationen, (1975)6, S. 189.

$93 \mathrm{Vgl}$. auch Mercer, The cycle of invasion and unification in the Western Sahara, in: African Affaires, (1976)301, S. 498-510(508f.). 


\section{Der Streitbeilegungsversuch durch die Organisation der Afrikanischen Einheit}

\subsection{Die Behandlung des Sahara-Konflikts in der OAU}

Vornehmstes Ziel der OAU bei ihrer Gründung war die Dekolonialisierung Afrikas und die Gewährleistung der Unabhängigkeit der dekolonisierten Gebiete. Die Politik der OAU stützte sich dabei von Beginn an auf die Prinzipien der Unverletzlichkeit kolonialer Grenzen und des Selbstbestimmungsrechts der Völker ehemaliger Kolonien. ${ }^{94}$ Solange es in der Westsahara um die Dekolonialisierung von Spanien und das allseits geforderte Selbstbestimmungsrecht für die Sahraouis ging, wies die Haltung der OAU in dieser Frage gegenüber gleichartigen Dekolonialisierungsfällen keine Besonderheit auf. Die Probleme der OAU begannen, als 1976 die drei Jahre zuvor gegründete Frente POLISARIO die offizielle Anerkennung als Befreiungsbewegung beantragte und Marokko hiergegen protestierte.

Im Januar 1976 hatte das OAU-Befreiungskomitee in Maputo (Mosambik) einem Bericht zugestimmt, der die F. POLISARIO als die "einzig legitime Befreiungsbewegung der sogenannten Spanisch-Sahara, die für die Unabhängigkeit des Landes kämpft", anerkennt. Die daher vom Befreiungskomitee im Februar empfohlene Anerkennung durch die OAU wurde jedoch von der Ministerkonferenz in Addis Abeba im März 1976 mit 21:9 Stimmen bei 17 Enthaltungen abgelehnt. ${ }^{95}$ Die ablehnende Haltung ist überwiegend auf die Drohung Marokkos und Mauretaniens, die sich aus dem Befreiungskomitee bereits demonstrativ zurückgezogen hatten, zurückzuführen, auch aus der OAU auszutreten, wenn die F. POLISARIO anerkannt würde. Nach der Proklamation der 'Demokratischen Arabischen Republik Sahara (DARS)، im Februar 1976 stand es im Belieben jedes einzelnen afrikanischen Staates, den Staat 'Sahara' anzuerkennen. Das Befreiungskomitee verabschiedete 1976 allerdings noch einmal eine Resolution, die der Bevölkerung der Westsahara das Recht auf Selbstbestimmung zubilligt.

Auf der die 13. OAU-Gipf elkonferenz in Port-Luis (Mauritius) vorbereitenden Ministerkonferenz wurde 1976 mit deutlicher Mehrheit ein Antrag verabschiedet, in dem "das unveräußerliche Recht des Volks der Spanischen Sahara auf Selbstbestimmung" und der "unmittelbare Abzug aller ausländischen Besatzungstruppen" gefordert wurde. Die anschließend tagende Gipfelkonferenz bestätigte dann zwar ebenfalls das Selbstbestimmungsrecht, ratifizierte jedoch nicht die Ministervorlage. Marokko und Mauretanien hatten für diesen Fall ihren Austritt angekündigt und waren zur Bekräftigung ihrer Absicht der Konferenz ferngeblieben. Sie waren aber diplomatisch am Tagungsort aktiv und veranlaßten das Gastgeberland Mauritius, den Vertretern der Sahara ein Aufent-

$94 \mathrm{Vgl}$. Wallerstein, The role of the Organization of African Unity in contemporary af rican politics, in: ElAyouty, Brooks (Hg.), Africa and International Organization, The Hague 1974, S. 18-28; Cervenka, The role of the O.A.U. in the peaceful settlement of disputes, ebenda, S. 48-68; Cervenka, The Organisation of African Unity and its Charter, 1968 sowie Kunig, a.a.O. (Anm. 2).

95 Vgl. Mercer, a.a.O. (Anm. 93), S. 509. 
haltsverbot aufzuerlegen. Die Versammlung der Staats- und Regierungschefs beschloß 1976, "eine Sondersitzung der Staats- und Regierungschefs unter Beteiligung des Volks der Westsahara" zu einem späteren Zeitpunkt einzuberufen. Dieser Beschluß gab zu erkennen, daß die OAU von einer nationalen Identität des sahraouischen Volks ausgeht, dessen Gebiet von fremden Truppen besetzt ist.

Die nächste Gipfelkonferenz 1977 in Libreville schlug die Abhaltung der Sondersitzung noch 1977 in Lusaka (Sambia) vor. Der OAU-Präsident Bongo (Gabun) sagte die Konferenz wegen Gefährdung der Teilnehmer durch angebliche Kämpfe an der sambisch-rhodesischen Grenze ab. ${ }^{96}$ Obwohl für 1978 noch einmal eine Sonderkonferenz vorgeschlagen wurde, hat sie bis heute nicht stattgefunden. Zwischenzeitlich tagte 1977 der Ministerrat in Lomé in Anwesenheit von Vertretern der F. POLISARIO. Das war für Marokko wieder Anlaß, seine Mitarbeit in der OAU nunmehr ganz aufzukündigen. Die Abwesenheit kam Marokko aber auch deswegen recht, weil es auf der Versammlung eine Verurteilung wegen Beteiligung an einer Aggression gegenüber Benin erwarten mußte. ${ }^{97}$ An der 14. Gipfelkonferenz in Libreville (Gabun) nahm Marokko dann doch wieder teil: Gabuns Regierung hatte den Vertretern der DARS keine Einreisegenehmigung erteilt. Auf der 15. Gipfelkonferenz in Khartum (Sudan) im Juli 1978 wurde eine rad hoc'-Kommission gebildet, das Komitee der 'Fünf Weisen` mit Nigeria, Guinea, Mali, Tanzania und Sudan. Der Arbeitsauftrag, dem Gipfel nach Anhörung der Parteien einen Bericht zu erstatten, wurde 1979 auf der 16. Gipfelkonferenz in Monrovia (Liberia), zu der die F. POLISARIO geladen worden war, erneuert; denn die 'Fünf Weisen hatten immer noch keinen Bericht erarbeitet. Die rad hoc'-Kommission hörte 1979 den Generalsekretär der F. POLISARIO an, was Hassan zum Vorwand nahm, keine Erklärungen vor den 'Fünf Weisen` abzugeben. Gleichwohl arbeitete die Kommission insofern im marokkanischen Interesse, als die OAU mit ihrer Beauftragung einen Weg gefunden hatte, sich auf längere Zeit nicht mehr entscheidungsfindend mit dem Problem befassen zu müssen.

Für die 17. Gipfelkonferenz in Freetown (Sierra Leone) im Juli 1980 war allgemein die Aufnahme der Republik Sahara in die OAU erwartet worden. Bereits ein Jahr zuvor hatten in Monrovia 23 von 50 Staaten ihre Aufnahmebereitschaft signalisiert. Neben Marokko hatten sich nur 3 Länder (Senegal, Gabun, Zaire) ablehnend ausgesprochen. ${ }^{98}$ Tatsächlich erreichte die DARS im Juli 1980 in Freetown mit 26 die Aufnahme befürwortenden Stimmen sogar die absolute Mehrheit der Mitgliedsstaaten und erfüllte damit die Aufnahmebedingungen nach Art. 28 der OAU-Charta. Marokko erklärte daraufhin, daß die DARS als nicht "unabhängiger souveräner Staat" kein OAU-Mit-

96 Nach Hinz, Gewalt gegen Selbstbestimmung, in: 3. Welt Magazin, (1977)11/12, S. 10f., tat Bongo alles, um den Gipf el zu verhindern; vgl. auch Damis, The moroccan-algerian conflict over the Western Sahara, in: The Maghreb Review, 4(1979)2, S. 49-57(55).

97 Vgl. Heinrich, Polisario pressure brings isolation for Morocco, Mauritania, in: New African Development, 11(1975)5, S. 429 und Kunig, a.a.O. (Anm. 2), S. 51.

98 Vgl. OAU-House politics and issues beyond, in: Africa, (1980)107, S. 18-22. 
glied werden könne. Wenn über diese Rechtsansicht keine Einigkeit bestünde, müsse mit einer 2/3-Mehrheit nach Art. 27 der Charta entschieden werden. Um dieses Problem zunächst beraten und begutachten zu können, wurde eine weitere Behandlung auf das 18. Gipfeltreffen 1981 vertagt.

Da die OAU bis 1981 nicht in der Lage war, entscheidenden Einfluß auf die Erfüllung ihrer Resolutionen auszuüben und den Streit in der Sahara beizulegen, wurde mit einer brisanten Sitzung des 18. Gipfeltreffens gerechnet. ${ }^{99}$ König Hassan II. kündigte auf einer Pressekonferenz vor Beginn der 18. Konferenz der Staats- und Regierungschefs konkrete Pläne für eine Friedenslösung in der Sahara an. ${ }^{100}$ Die den Gipfel vorbereitenden Konferenzen der Experten und der Minister beschlossen angesichts einer möglichen spektakulären Wende der marokkanischen Saharapolitik noch nicht über eine Einladung und damit offizielle Aufnahme der DARS in die OAU. Damit war ein weiterer diplomatischer Schachzug Marokkos gelungen.

Vor der Abreise nach Nairobi wiederholte Hassan, daß er einen Plan vortragen werde, mit dem die "Angelegenheit der Sahara" beendet werden könne. Mit einem Referendum wolle er die Zugehörigkeit der Sahara zu Marokko dokumentieren: "Nous avons tenus compte de la souplesse du droit international et du contexte af ricaine. Nous ne renoncerons, ce faisant, à aucun grain de sable de ce Sahara marocain pour lequel tant des nôtres ont sacrifié leur sang et qui a coûté beaucoup d'argent."101

Damit hat Hassan II. deutlich gemacht, daß ein von ihm vorgeschlagenes Referendum nicht das Selbstbestimmungsrecht berücksichtigen, sondern seine Sahara-Politik bestätigen lassen soll. Mit entsprechend zwiespältigen Gefühlen wurden die Vorschläge Marokkos auf der Konferenz aufgenommen. ${ }^{102}$ Am 26. 6. akzeptierte Hassan die vom 'Komitee der Sieben Weisen ${ }^{103}$ aufgestellten Forderungen nach einem überwachten Referendum in der Sahara. Die Gipfelkonferenz beauftragte diese rad hoc'-Kommission, mit den Konfliktparteien die Modalitäten für einen Waffenstillstand und für das Referendum bis Ende August 1981 auszuarbeiten. Es erging ein Ersuchen an die UN, zusammen mit der OAU eine Friedenstruppe in die Sahara zu entsenden und dort bis nach den ersten Wahlen zu stationieren. Damit bat die OAU erstmals direkt die UN in eigener Angelegenheit um Unterstützung. Die Resolution forderte von Marokko weder den Rückzug der Truppen, noch eine Ubergabe der Verwaltung an Neutrale. Marokko hatte zu erkennen gegeben, daß es eine solche Forderung nie akzeptieren würde. Die Resolution enthält auch nicht ausdrücklich den Namen der F. POLISARIO als direkt beteiligte Streitpartei. ${ }^{104}$ 
Beobachter der Konferenz sahen in der Resolution einen Erfolg des marokkanischen Herrschers, der mit einem vagen Angebot Zeit gewonnen und die letztendliche Entscheidung über die Mitgliedschaft der DARS als 51. OAU-Mitglied verhindert habe. ${ }^{105}$ Es ist aber hervorzuheben, daß Marokko nach über 6 Jahren Kriegsführung erstmals zugegeben hat, daß das Sahara-Problem ein Entkolonialisierungsproblem ist; denn erstmals nach dem Rückzug der Spanier soll mit Zustimmung Marokkos der Bevölkerung der Westsahara mit dem Referendum die Möglichkeit der Selbstbestimmung eingeräumt werden.

Die F. POLISARIO hat im Anschluß an die Konferenz ihr Einverständnis für die Durchführung eines Referendums gegeben, obwohl sie in der Vergangenheit auch betont hat, daß das Volk der Sahara mit der Gründung der DARS und der Wahl einer Regierung seinen Willen bereits zum Ausdruck gebracht habe. Sie stellt für die Durchführung des Referendums folgende Bedingungen:

1. Direkte Verhandlungen zwischen der F. POLISARIO und der marokkanischen Regierung über einen Waffenstillstand,

2. Rückzug der marokkanischen Armee aus der Westsahara,

3. Abzug der marokkanischen Verwaltung,

4. Stationierung der marokkanischen Truppen $150 \mathrm{~km}$ hinter der Grenze zwischen der Sahara und Marokko,

5. Rückkehr der sahraouischen Flüchtlinge in ihre Heimatstädte und -dörfer,

6. die Einrichtung einer vorübergehenden internationalen Verwaltung unter Mitwirkung der UN und der OAU und in Zusammenarbeit mit der Regierung der DARS

7. Tätigkeit dieser internationalen Verwaltung für mindestens drei Monate,

8. Freilassung aller sahraouischen Gefangenen einschließlich Frauen und Kindern aus den marokkanischen Gefängnissen und Konzentrationslagern,

9. Stationierung einer UN-Friedenstruppe entlang der marokkanisch-sahraouischen Grenze für die Dauer der Durchführung des Referendums. ${ }^{106}$

Als unverzichtbar wurde von ihr auch erklärt, daß Marokko bilaterale Verhandlungen direkt mit der F. POLISARIO unter dem Schutz internationaler Organisationen akzeptieren müsse. Hassan hatte im Juli 1981 noch einmal erklärt, er werde mit der F. POLISARIO, die "niemals für die afrikanische Gemeinschaft existiert" habe, nicht verhandeln. ${ }^{107}$

Das Komitee hatte eine schwierige Situation zu meistern: Neben den genannten sich gegenüberstehenden Forderungen der Streitparteien mußte es den kompliziertesten

105 Siehe FR 29.6. 1981; NZZ 30.6. 1981; FAZ 1. 7. 1981 und afrique-asie No. 245, 3. 8. 1981, S. 16; vgl. auch Plinke, Der Traum von Großmarokko scheint ausgeträumt, Hannoversche Allgemeine Zeitung 3. 7. 1981 und Baumhögger, Die OAU am Scheideweg, in: Dt. Ubersee-Institut (Hg.), Jahrbuch Dritte Welt 1, München 1983, S. 221-236(225f.).

106 Zit. in: Sahraoui setzen auf UNO-Kontrolle, Die Neue 27. 8. 1981.

107 Le monde und EL PAIS 4. 7. 1981. 
Streitpunkt, die Festlegung der Teilnehmer am Referendum, befriedigend lösen. Wie sollte beispielsweise die Nationalität der grenzüberschreitenden Nomaden festgestellt werden? Die F. POLISARIO erklärt den spanischen Censo von 1974 für ungültig. Sie will die Sahraouis, die in den Lagern in Algerien im Exil leben, an der Abstimmung beteiligen. Scharf verurteilt wurde von ihr die marokkanische Verhaftungs- und Verschleppungswelle gegen Sahraouis, insbesondere in den Garnisonstädten. ${ }^{108}$ Außerdem weist sie daraufhin, daß in der Westsahara nur noch etwa 45000 Sahraoui verblieben sind, während 70000 marokkanische Soldaten stationiert worden seien und Marokko in einem noch fortdauernden Prozeß bisher 60000 Einwohner neu angesiedelt habe. ${ }^{109}$ Demgegenüber fordert Hassan die Verhinderung der Beeinflussung des Referendums durch 'Ausländer', womit er die sich seiner Ansicht nach aus Algeriern, Mauretaniern und Maliern zusammensetzende F. POLISARIO und die Flüchtlinge in Algerien meint. Grundlage für die Teilnahme am Referendum solle der spanische Censo ' 74 sein, dessen Ergebnis zufolge nur etwa 95000 Menschen in der Sahara wohnen sollten. Damit steht der Herrscher allerdings im Widerspruch zu seinen eigenen Angaben vor den UN im Jahre 1966, als er den Abzug der Spanier aus dem von 150000 Sahraoui bewohnten Gebiet verlangte. ${ }^{110}$

Ende August tagte schließlich nach Anhörung der Parteien das mit allen Vollmachten von der OAU ausgestattete Komitee hinter verschlossenen Türen, veröffentlichte den "3-Punkte-Plan der OAU" und rief zum Waffenstillstand auf. Die durch das Referendum zu beantwortende Frage sollte lauten: 1. Anschluß an Marokko oder 2. unabhängiger, souveräner Staat.

Die drei Vorschläge des Komitees waren: 1. Die einzurichtende provisorische Verwaltung hat die zivile, militärische und polizeiliche Kompetenz zur Organisierung des Referendums unter Einschaltung der marokkanischen Verwaltung. 2. Das Referendum steht unter Beobachtung von OAU und UN. 3. Stimmberechtigt sind nur die im Zensus 1974 aufgezählten Personen sowie die Bewohner der Flüchtlingslager, soweit sie der UN-Kommissar für Flüchtlinge anerkannt hat (circa 230 000). ${ }^{111}$

Das Komitee hat mit diesem Programm Kompromisse angeboten. Zugunsten Marokkos ist der Abzug von Militär und Verwaltung nicht offiziell gefordert worden, wenngleich dazu vom Komitee mündlich aufgefordert wurde. Gleichzeitig wird aber in der Begründung der illegale Charakter der marokkanischen Anwesenheit betont, was der F. POLISARIO entgegenkommt. Die Verhandlungen zwischen den beiden kriegführenden Parteien sollen indirekt verlaufen. Die Truppen müssen kaserniert werden. Marokko war verstimmt, weil die Referendumsfrage die Unabhängigkeit der Sahara offen ließ, weil eine provisorische internationale Verwaltung zu einem Machtzuwachs

111 EL PAIS 27. 8. 1981. 
gegen Marokkos zukünftige Interessen führen könnte und weil gegen marokkanisches Verfassungsrecht die Stimmberechtigung auch den 18jährigen und nicht erst den 21 jährigen eingeräumt werden sollte. ${ }^{112}$

Unglücklich ist an dem Ergebnis, daß die Flüchtlinge in ihr Gebiet zurückkehren sollen, obwohl dort noch die marokkanische Verwaltung tätig bleiben kann, vor der die Sahraouis geflüchtet waren. Selbst wenn unter internationalem Schutz Úbergriffe weitgehend vermieden werden können, ist zweifelhaft, ob ein "günstiges Klima" für die Durchführung eines Selbstbestimmungsentscheids geschaffen werden kann, der auf der Grundlage völliger Freiheit demokratisch und unvoreingenommen durchgeführt werden sollte. ${ }^{113}$ In diesem Sinne fordern auch die UN-Resolutionen "to create a favourable climate for the referendum to be conducted on an entirely free, democratic and impartial basis. "114 Diese Forderung nach einem "günstigen Klima" wurde von dem UN-Sonderausschuß für Aden im Juli 1963 entwickelt: "Die Ausübung des Selbstbestimmungsrechts muß in Form einer sobald als möglich abzuhaltenden Befragung der gesamten Bevölkerung auf der Grundlage des uneingeschränkten Erwachsenenstimmrechts und unter Beachtung der Menschenrechte und Grundfreiheiten erfolgen. "115

Algerien veröffentlichte ein Memorandum, in dem es ebenfalls forderte: Waffenstillstandsverhandlungen zwischen beiden Parteien, Einsetzung einer UN-Friedenstruppe, Installierung einer provisorischen Verwaltung, Volkszählung in der Sahara nach Rückkehr der Flüchtlinge und Organisierung des Referendums. ${ }^{116}$

Die marokkanische Position wurde nach Vorlage der Referendumspläne als geschwächt angesehen. Es war daher zu erwarten, daß Marokko weiterhin alles versuchen würde, um die Durchführung des Referendums hinauszuzögern oder gar zu verhindern. Die Kämpfe um die marokkanische Garnison Guelta Zemmour im September 1981, in dessen Verlauf mehrere Flugzeuge zerstört wurden, heftige Panzerkämpfe zur Zerstörung der Stadt führten und hunderte Soldaten fielen, ${ }^{117}$ ließen Waffenstillstandsverhandlungen und Vorbereitungen für das Referendum in weite Ferne rücken. Der Generalsekretär der OAU, Edem Kodjo, erkundete in Rabat Möglichkeiten für die Fortentwicklung des Friedensprozesses und besuchte danach auch erstmals die Sahara. Der Generalsekretär der OAU erklärte, als ein Scheitern der OAU-Bemühungen um einen Waffenstillstand of fensichtlich wurde, die Aufnahme der Republik Sahara in die afrikanische Staatengemeinschaft als ihr 51. Mitglied. Kodjo konnte sich seinen Kritikern gegenüber in einem Memorandum ${ }^{118}$ auf Art. 28 der OAU-Charta berufen, in dem es heißt, daß die Entscheidung eines jeden Mitgliedsstaats über das Beitrittsgesuch eines

112 EL PAIS 2. 9. 1981.

113 Vgl. Rabl, a.a.O. (Anm. 28), S. $416 \mathrm{ff}$.

114 Res. 2229(XXI), 2354(XXII), 2428(XXIII), 2592(XXIV) und 2711(XXV).

115 Zit. bei Rabl., a.a.O. (Anm. 28), S. 416.

116 EL PAIS 3. 9. 1981.

117 Vgl. Le monde 20., 22. und 28. 10. 1981.

118 Abgedruckt in jeune afrique, No. 1147/1148, 29. 12. 1982/5. 1. 1983, S. 55 ff. 
Staats dem Generalsekretär mitzuteilen ist. Dieser hat satzungsgemäß nach Eingang von 26 zustimmenden Voten, der absoluten Mehrheit der Mitglieder, den Aufnahmebeschluß bekanntzugeben. Die Charta statuiert demnach eine Rechtspflicht des Generalsekretärs, bei mehrheitlicher Befürwortung eines Aufnahmeersuchens alle Mitglieder über dieses Ergebnis zu informieren und dadurch das Aufnahmeverfahren formell abzuschließen. Da 26 afrikanische Staaten die Demokratische Arabische Republik Sahara anerkannt und eine Aufnahme in die OAU befürwortet hatten, war Kodjo zu diesem administrativen Schritt verpflichtet, den er seit 1980 gegen die Charta hinausgezögert hatte.

Wenngleich der Satzungswortlaut sogar schon eine einfache Mehrheit der OAU-Mitglieder für eine Aufnahmeentscheidung genügen läßt, zweifelte Marokko die Wirksamkeit der Aufnahmeentscheidung an. Da nach Art. 4, 28 der Charta nur unabhängige souveräne Staaten der OAU beitreten könnten, sei zunächst die Staatsqualität der DAR Sahara festzustellen. Diese Feststellung sei aber eine Auslegung eines Charta-Begriffs und müsse daher nach Art. 27 mit einer 2/3-Mehrheit der Mitglieder entschieden werden. Diese Rechtsauffassung Marokkos und einiger befreundeter Staaten ('Ablehnungsfront') ist nicht haltbar. Zum einen folgt der die Aufnahmemodalität regelnde Art. 28 in der Satzungssystematik dem Art. 27, der sich demnach of fensichtlich auf Dissense über vorangehende Regelungen in der Charta beziehen soll. Da eingewandt werden könnte, daß der Begriff des unabhängigen souveränen Staats bereits in Art. 4 verwendet wird, ist für ein Verständnis von Art. 28 der Wortlaut bedeutsam. Er läßt, gerade im bewußten Gegensatz zu den Mehrheitsregelungen in Art. 10 Ziff. 2 und 4, Art. 12 Satz 3, Art. 14 Ziff. 3 und Art. 27, für eine Entscheidung über die Aufnahme eines neuen Mitglieds die Zustimmung der einfachen Mehrheit genügen. Da jeder unabhängige souveräne af rikanische Staat nach Art. 4 berechtigt sein soll, Mitglied der Organisation zu werden, regelt Art. 28 gerade die Entscheidungsfindung innerhalb der OAU auch hinsichtlich einer Auslegung von Art. 4. Schon die einfache Mehrheit soll entscheiden können, ob der die Aufnahme Ersuchende ein unabhängiger souveräner Staat ist und damit berechtigt die Mitgliedschaft erwerben kann. Eine Auslegung dieser speziellen Frage wird demnach derzeit bei 50 oder 51 Mitgliedern mit einer Mehrheit von 26 Stimmen positiv entschieden. Eine Anwendung von Art. 27 gegen den Wortlaut von Art. 28 ließe die Motive der Satzungsgeber außer acht und die Regelung dieses Artikels leerlaufen.

Sofern Marokko mit der 'Ablehnungsfront ${ }^{119}$ diese Regelung mißachtet, bewegt es sich außerhalb des von der OAU-Satzung gesetzten Rechts. Das marokkanische Beharren auf seinem Rechtsstandpunkt kommt einer politischen Erpressung der afrikanischen Staatenmehrheit gleich. ${ }^{120}$ Marokko hat die OAU unter Druck gesetzt, die Aufnahmeentscheidung zu revidieren, indem es die 'Ablehnungsfront` zu einem Boykott der 38. 
und 39. Ministerratstagung 1982 in Äthiopien und Libyen und der OAU-Arbeitsministerkonferenz veranlaßte. Den Höhepunkt der von Marokko provozierten Krise bildete das Scheitern der nach Tripolis (Libyen) zum 5.-8. 8. 1982 einberufenen 19. Gipfelkonferenz. Selbst der von der Republik Sahara angebotene Kompromißvorschlag, auf Einnahme ihres Sitzes in der Versammlung vorübergehend zu verzichten, konnte das nach Art. 10 Ziff. 4 der Charta erforderliche 2/3-Anwesenheitsquorum nicht bewirken.

Die starre Haltung der 'Ablehnungsfront ' wird auch auf das politische, von den USA geförderte ${ }^{121}$ Motiv zurückzuführen sein, eine Gipfelkonferenz in Libyen und damit die turnusmäßige UUbernahme des OAU-Vorsitzes durch den umstrittenen Revolutionsführer Kadhafi zu verhindern. Dafür spricht auch, daß trotz weiterer Kompromißangebote in der Sahara-Frage und eine erneut auf November 1982 terminierte Gipfelkonferenz nicht zustande kam. Das Scheitern von Tripolis II wurde mit dem Tchad-Konflikt begründet. ${ }^{122}$ Zutreffend sieht Baumhögger in der Tchad-Frage den Vorwand, nicht aber den eigentlichen Grund für das Scheitern, "der vielmehr darin zu sehen sein dürfte, daß es Marokko gelang, die eigenen Reihen geschlossen zu halten - zur Durchsetzung einer Strategie einer möglichst nachhaltigen Lähmung der OAU “. ${ }^{123}$

Entstanden war nunmehr eine Situation, auf die die OAU-Charta nicht zugeschnitten ist; ein Ausweg war formell nicht geregelt. Die Satzung sieht mindestens jährliche Gipfeltreffen vor, damit zumindest der Haushalt verabschiedet, Vorsitzender und Generalsekretär gewählt und der Ort der nächsten Konferenz der Staats- und Regierungschefs festgelegt werden können.

Die in Tripolis im Herbst 1982 versammelten 31 Delegationen hatten ohne OAU-Legitimation ein "Komitee der 12 " eingerichtet, das sich um die Einberufung einer Gipfelkonferenz zum 6. Juni 1983 nach Addis Abeba (Æ̈thiopien) bemühte. Ebenso unternahm das von 9 auf 21 Staatenvertreter erweiterte geschäftsführende Büro der 18. Gipfelkonferenz von Nairobi Anstrengungen für die Eröffnung eines Gipfels. ${ }^{124}$ Als der sahraouische Außenminster Hakim im Interesse der Einheit Afrikas "freiwillig und zeitweilig" auf eine Teilnahme am Gipfeltreffen verzichtete, konnte am 8. Juni 1983 nach tagelangen Verhandlungen vor den Türen des Sitzungssaals die 19. Versammlung der Staats- und Regierungschefs zusammentreten. Außer der sahraouischen war der Eröffnung nur noch die libysche Vertretung ferngeblieben. Zum Nachfolger des Vorsitzenden Moi wurde der äthiopische Staatschef Mengistu Haile Mariam gewählt.

Am 11. Juni 1983 verabschiedete die Konferenz eine Resolution, in der Marokko und die F. POLISARIO aufgefordert werden, "direkte Verhandlungen zu unternehmen, um zu einem Waffenstillstand zu gelangen, der die notwendigen Bedingungen für ein friedliches und gerechtes Referendum im Hinblick auf die Selbstbestimmung des Volks in der

121 Nach Berichten der 'Newsweekı vom 3. 10. 1983 (vgl. taz 5. 10. 1983) sind CIA-Pläne, den libyschen Revolutionsführer Kadhafi zu liquidieren, erst am Einspruch des amerikanischen Kongresses gescheitert.

122 Ausführlich Baumhögger, a.a.O. (Anm. 105), S. $230 \mathrm{ff}$.

123 Ebenda, S. 231.

124 Vgl. NZZ 9. 6. 1983 und Archiv der Gegenwart 12.6. 1983, 26711 A. 
Westsahara schafft."125 Zur nächsten Gipfelkonferenz in Conakry (Guinea) soll die Sahara-Kommission bereits über die Ergebnisse eines Referendums, das bis Ende 1983 durchgeführt sein sollte, berichten. Die Resolution nennt die Konfliktparteien, die in direkte Verhandlungen eintreten sollen, erstmals beim Namen. Zwar erwähnt sie nicht die Regierung der Republik Sahara, so aber doch die F. POLISARIO. Ferner soll das Referendum ohne administrative oder militärische Behinderung stattfinden, ohne daß aber wie früher eine Kasernierung der Truppen und der Einsatzeiner neutralen Interimsverwaltung gefordert werden. Die Vereinten Nationen werden gebeten, sich an einer OAU-Friedenstruppe zu beteiligen.

Da bis zum Ende 1983 das geforderte Referendum nicht abgehalten werden konnte, weil Hassan sich weiterhin einer direkten Verhandlung zwischen den Konfliktparteien widersetzt, liegt die Behandlung des Konflikts wieder in den Händen der Versammlung der Staats- und Regierungschefs auf der 20. Gipfelkonferenz. Die Republik Sahara hat angekündigt, nicht ein weiteres Mal auf ihre Mitgliedschaftsrechte verzichten zu wollen. Ihre Regierungsvertreter werden andererseits darauf vertrauen müssen, daß ihnen nicht von Guinea die Einreisevisa verweigert werden, was bereits einmal geschehen ist. Marokko wird es nicht wieder gelingen, eine 'Ablehnungsfront ' von mindestens 16 Staaten zusammenzubekommen, um die Versammlung erneut a m fehlenden Quorum scheitern zu lassen. Sogar Senegals Präsident Diouf, maßgeblich an der Formulierung der Sahara-Resolution beteiligt, ist auf Distanz zu Marokko gegangen und hatte in Addis-Abeba den Präsidenten der Republik Sahara, Abdelaziz, zu einem Gespräch aufgesucht. ${ }^{126}$

Wird der 20. Gipfel keine greifbare Lösung des Sahara-Konflikts finden, ist eine Verstärkung der Kampfhandlungen in dem von Marokko besetzten Sahara-Teil zu befürchten. Die F. POLISARIO hatte bereits im Juli, August und September 1983 nach längerer Kampfpause im Anschluß an einen Anfang 1982 verkündeten einseitigen Waffenstillstand wieder Offensivangriffe gegen Stellungen in Südmarokko geführt, ${ }^{127}$ bei denen über 500 marokkanische Soldaten fielen, und erhebliche Mengen einschließlich zahlreicher Raketenwerfer und Panzer zerstört oder erbeutet wurden.

\subsection{Möglichkeiten und Grenzen einer Streitbeilegung durch die Organisation der Afri- kanischen Einheit}

Die OAU hat nicht vermocht, den Frieden im Nordwesten Afrikas nach dem Abzug Spaniens aus der Westsahara zu sichern. Der Ubergang der Kämpfe gegen die Kolonialmächte Spanien und Frankreich zu einem - nunmehr seit 8 Jahren anhaltenden - offenen Krieg zwischen Afrikanern hat die Grenzen der Fähigkeit der OAU deutlich gemacht, in lokale Konflikte friedenserhaltend und -stiftend einzugreifen.

125 Der französische Wortlaut der Resolution ist abgedruckt in Le Soleil (Dakar), 13. 6. 1983.

126 NZZ 15.6. 1983.

127 FR 12. 7. 1983; taz 26. 8. 1983; Archiv der Gegenwart 14. 7. 1983, 26804. 
Die OAU hat mit der Mehrheit ihrer Mitglieder den Westsahara-Konflikt stets als ein das Prinzip des Selbstbestimmungsrechts von Kolonialvölkern berührendes Problem gesehen. Sie ist nicht der marokkanischen Auffassung gefolgt, wonach der Dekolonialisierungsprozeß in der ehemaligen Spanisch-Sahara mit der wirksamen Eingliederung in das marokkanische Staatsgebiet abgeschlossen sei. In einen dementsprechend als Sezessionsbewegung zu qualifizierenden Kampf der F. POLISARIO hätte sich die OAU auch nicht eingemischt. ${ }^{128} \mathrm{Da}$ die Befreiungsbewegung in der Sahara aber nicht gegen die Teilbesetzung des Gebiets durch die alte Kolonialmacht kämpft, ${ }^{129}$ sondern gegen einen af rikanischen Nachbarstaat, ist die für die OAU wohl einmalige Situation eingetreten, daß ein af rikanischer Staat, der Mitglied der OAU ist, als quasi-Kolonialmacht betrachtet werden muß, gegen den sich die OAU-Ziele auf Dekolonialisierung des Kontinents beziehen; "es geht also 'noch' um die erstmalige Ausübung des Selbstbestimmungsrechts". ${ }^{130}$ Auf diese befremdliche Konstellation hatte zuerst der Vertreter Zimbabwes auf der 17. Gipf elkonferenz in Freetown hingewiesen.

Aufgrund dieser Situation kann diejenige Auffassung auf diesen Konflikt bezogen werden, nach der "die Unterdrückung eines Befreiungskampfs durch die Kolonialmacht als Verstoß gegen das Gewaltverbot und damit als unzulässig angesehen wird, während umgekehrt der Träger des Selbstbestimmungsrechts auf diesem Gebiet, die Befreiungsbewegung, völkerrechtlich legitimiert ist, auch mit militärischer Gewalt gegen die Kolonialmacht vorzugehen ". ${ }^{131}$ Das Problem rechtmäßiger Gewaltanwendung kann also auch unter den Gesichtspunkten untersucht werden, die im Zusammenhang mit dem Selbstbestimmungsrecht der im antikolonialen Freiheitskampf stehenden Völker entwikkelt wurden. ${ }^{132}$

Eine allgemein anerkannte Definition für 'Aggression ‘ ist nicht vorhanden. Unbestritten ist jedoch, daß das bewaffnete Eindringen in das Gebiet eines anderen Staats eine Aggression darstellt. ${ }^{133}$ Wenn bereits der Widerstand durch die (ehemalige) Kolonialmacht gegen das Selbstbestimmungsrecht des (ehemaligen) Kolonialvolks als Aggression im völkerrechtlichen Sinn bezeichnet wird, ${ }^{134}$ ist das marokkanische Verhalten, der Einzug mit Militärgewalt in benachbartes Gebiet ohne Rechtfertigungsgrund ebenfalls eine Aggression. Diskussionsfähig bleibt dann allenfalls die Frage, ob bereits der mit militärischem Einmarsch einhergehende 'Grüne Marsch' eine solche Aggression gegenüber der sahraouischen Bevölkerung darstellte, da er noch stattfand, als die Kolonial-

Vgl. Kunig, a.a.O. (Anm. 25), S. 272 ff. und - in Bezug auf Biafra - Ansprenger, Die OAU auf dem langen Weg von der Pluralität zum Pluralismus, in: Festschrift für Fraenkel, Hamburg 1973, S. 280-330 (287).

Völkerrechtliche Fragen eines solchen Konflikts behandelt Bothe, Völkerrechtliche Aspekte des AngolaKonflikts, in: Zeitschrift für ausländisches öf fentliches Recht und Völkerrecht, (1977)37, S. 572-603.

Kunig, a.a.O. (Anm. 25), S. 189f. Bothe, a.a.O. (Anm. 129), S. 576.

Ausführlich Bothe, a.a.O. (Anm. 129), S. 575 mit Anm. 16.

Wittig, Der Aggressionsbegriff im internationalen Sprachgebrauch, in: W. Schaumann (Hg.), Völkerrechtliches Gewaltverbot und Friedenssicherung, Baden-Baden 1971, S. 33-72(46).

So Wittig, ebenda, S. 59. 
macht Spanien in der Westsahara präsent war. ${ }^{135}$ Letztendlich kommt es darauf heute nicht mehr an, da Marokko jedenfalls nach dem 27. 2. 1976 eine permanente Aggression ausübt.

Nach Wildhaber ${ }^{136}$ wäre nicht allein die Selbstverteidigung eines Volks, hier des sahraouischen, gegen eine bewaffnete Aggression völkerrechtlich zulässig, sondern auch eine kollektive Selbstverteidigung unter Berufung auf Art. 51 der UNO-Satzung und auf Naturrecht. Es könnte demnach sogar ein drittes Land den Sahraouis militärisch zu Hilfe kommen, ohne gegen Völkerrecht zu verstoßen. Diese Auffassung begründet in anderem Zusammenhang auch Bothe: "Wenn man als Inhalt des Selbstbestimmungsrechts ansieht, daß es sich ohne auswärtige militärische Intervention verwirklichen können soll, dann erscheint die Folgerung nicht unangemessen, daß dem einmal rechtswidrig erfolgten Eingriff in die Ausübung des Selbstbestimmungsrechts rechtmäßig durch einen dritten Staat entgegengewirkt werden kann, um die störungsfreie Ausübung des Selbstbestimmungsrechts wieder zu ermöglichen, um die Verfälschung der Ausübung des Selbstbestimmungsrechts wieder auszugleichen. "137 Bisher hat aber weder die Republik Sahara zum direkten Eingreifen aufgerufen, noch ist ihr dies von dritter Seite angeboten worden.

Eine Untersuchung des Unvermögens, diesen Regionalkonflikt zu beenden, hat den subjektiven Willen und die objektiven Fähigkeiten der OAU, friedensstiftend bei innerafrikanischen Auseinandersetzungen zu wirken, zu berücksichtigen. Die Möglichkeiten der OAU eines 'peaceful settlement of disputes` ergeben sich aus ihrer Charta, für deren Verständnis ihre Entstehungsgeschichte zu beachten ist.

In Anlehnung an die Charta der Vereinten Nationen hat die Konferenz der Unabhängigen Staaten in Akkra im April 1958 in der "Resolution on the Exchange of Views on Foreign Policy" die folgenden Mittel zur friedlichen Streitbeilegung aufgezählt: "Settlement of all international disputes by peaceful means, such as negotiations, conciliations, arbitration or judicial settlement as well as other peaceful means of the parties' own choice". Auch die aus der Brazzaville-Gruppe hervorgegangene Union der Staaten Afrikas und Madagaskars sah sich aufgrund eines Zwischenfalls zwischen den Mitgliedern Gabun und Kongo/Brazzaville veranlaßt, "a permanent Commission for the conciliation of disputes and a Court of Arbitration" vorzuschlagen und ein Gutachten eines Richters des Internationalen Gerichtshofs, des Senegalesen M. Forster, über die Möglichkeiten eines schiedsgerichtlichen Verfahrens einholen zu lassen. Die Intention, die Regeln der friedlichen Streitbeilegung vertraglich zu verankern, verfolgte auch die Inter-African and Malagasy Organisation', deren Mitglieder darin übereinstimmten, als Bestandteil ihrer Charta einen Vertrag zwischen den Signartarstaaten über die Einrichtung einer "Permanent Conciliation Commission" zu schließen. Kam in den

136 Gewaltverbot und Selbstverteidigung, in: Schaumann (Hg.), a.a.O. (Anm. 133), S. 147-172, passim.

137 A.a.O. (Anm. 129), S. 584f.: a.A. wohl Neuhold, Internationale Konflikte - verbotene und erlaubte Mittel ihrer Auslegung, Wien New York 1977, S. 142f.,191ff. 
Chartas und Resolutionen dieser Gruppe der Wille zum Ausdruck, die Friedenssicherung und Streitbeilegung zum vordringlichen Anliegen zu machen und zu institutionalisieren, so findet sich in den Chartas der Casablanca-Gruppe von 1961 und der "Union of African States" nichts dergleichen. Zwar strebten die Staaten einen afrikanischen Bundesstaat mit dem Ziel an, "to preserve and consolidate identity of view and unity of action in international affairs", doch waren die zum Ausdruck gebrachten politischen Prioritäten insofern von auffälliger Kurzsichtigkeit, als sich ihr Vereinigungsmotiv darauf beschränkte, "to liquidate colonialism and neo-colonialism in all their forms and, equally, to strive to rid the African Continent of political and economic intervention". Sie ignorierten damit die ideologischen Differenzen zwischen den unabhängigen Staaten Afrikas. ${ }^{138}$

Die bei der Frage nach der friedenstiftenden Rolle eines af rikanischen Staatenbündnisses sichtbar werdenden unterschiedlichen Prioritäten spiegeln sich schließlich auch in der Charta der OAU wieder. Die Beilegung von Streitigkeiten wird in ihr als Prinzip des Umgangs der Mitglieder untereinander angesprochen. Diesbezüglich weisen Wortlaut und Regelungsbereich der OAU-Charta aus, daß ihrer Verabschiedung ein Kompromiß zwischen den Gründungsmitgliedern bzw. zwischen rivalisierenden ideologischen Blökken (z. B. der Brazzaville- und der Casablanca-Gruppe) vorausgegangen sein muß.

So beinhaltet dieser Kompromiß etwa, daß die OAU von Beginn an von den Mitgliedsstaaten nicht als friedenstiftende Institution betrachtet, sondern sie (nur) als ein Instrument der Förderung von Einheit und Solidarität der afrikanischen Staaten gesehen wurde. Das Schlagwort von der Förderung von Einheit und Solidarität umfaßt dabei - erschöpfend - die OAU-Ziele der Dekolonisation Afrikas und die Ausmerzung von Rassendiskriminierung sowie die einer Verbesserung des allgemeinen Lebensstandards, die der Verteidigung der Souveränität, territorialen Integrität und Unabhängigkeit. Diese Ziele werden von der Charta zum Ausdruck gebracht. Dementsprechend sprach der Generalsekretär von der OAU auch nur als einem "Instrument der Bef reiung, der Entwicklung und des Fortschritts in Afrika". ${ }^{139}$ Das Ziel, Frieden zwischen den Mitgliedstaaten zu sichern und herbeizuführen, wird unter Art. 2 I der Charta nicht ausdrücklich genannt. In Art. 2 Ziffer 4 wird das Prinzip der friedlichen Beilegung von Streitigkeiten durch Verhandlung, Vermittlung und Schlichtung genannt. Als ein Prinzip der OAU appelliert es an die einzelnen Mitgliedsstaaten, verpflichtet aber nicht die Organe der OAU, friedenstiftend tätig zu werden.

Durch Art. 19 Satz 2 hat die OAU als Organ die "Commission of Mediation, Conciliation und Arbitration" geschaffen. Auch Art. 19 verpflichtet die Mitgliedstaaten untereinander zur friedlichen Streitbeilegung. Es legt den Mitgliedstaaten auch nicht die Rechtspflicht auf, die Kommission zur Streitbeilegung anzurufen. ${ }^{140}$ Arbeitsbedingun-

138 Vgl. Cervenka, in: El-Ayouty, Brooks (Hg.), a.a.O. (Anm. 94), S. 48ff.

139 Zit. ebenda, S. 53.

140 Vgl. Kunig, a.a.O. (Anm. 25), S. 139 mit Anm. 227. 
gen und Zusammensetzung der Kommission werden durch ein von der Versammlung der Staats- und Regierungschefs bestätigtes Protokoll festgelegt, das nach Satz 3 zum Bestandteil der Charta erklärt wurde. Dieses "Protocol on the Commission of Mediation, Conciliation and Arbitration" wurde im Juli 1964 von der Versammlung der Staats- und Regierungschefs auf ihrer Konferenz in Kairo angenommen. ${ }^{141} \mathrm{Nach}$ ihm verfügt die OAU über drei Möglichkeiten zu friedlicher Streitbeilegung: Vermittlung, Aussöhnung und Schiedsspruch.

Im Unterschied zu Artikel 33 der Charta der Vereinten Nationen, nach der der Sicherheitsrat die streitenden Parteien auffordern kann, die Streitigkeit durch Verhandlung, Untersuchung, Vermittlung, Vergleich, Schiedsspruch, gerichtliche Entscheidung, Inanspruchnahme regionaler Einrichtungen oder Abmachungen oder durch andere friedliche Mittel eigener Wahl beizulegen, fehlt in der Charta der OAU einschließlich eingeführtem, genannten Protokoll die Regelung einer Streitbeilegung durch gerichtliche Entscheidung. Von der Einrichtung eines Gerichtshofs, der sich mit dem "Völkerrecht der Staaten Afrikas" bzw. den Rechtsbeziehungen zwischen den OAU-Mitgliedern auseinanderzusetzen hätte, sahen die Gründungsmitglieder ab. Da auch die Möglichkeiten des Rückgriffs auf UNO-Organe bzw. des Anrufens der UNO durch die OAU nicht vorgesehen ist, ${ }^{142}$ bleibt die Frage nach dem Charakter der "Rechtsprechung" der in Art. 19 vorgesehenen Kommission. Die Empfehlungen, Resolutionen oder Schiedssprüche dieser Kommission haben nach dem Wortlaut der Charta (einschließlich Protokoll) ipso facto keine Bindungswirkung ('compulsory or optional jurisdiction of the commission (). ${ }^{143}$ In der Praxis besteht dementsprechend auch Ubereinstimmung, daß in dem Fall, daß eine Partei sich weigert, sich der Kommissionsentscheidung zu unterwerfen, die Streitfrage als politische Sache behandelt wird und zur Beratung an den Ministerrat (zurück-)verwiesen wird. Diese Konstruktion hat dazu geführt, daß die Kommission nach ihrem erstmaligen konstituierenden Zusammentreffen in der Vergangenheit von keiner streitführenden Partei angerufen wurde. ${ }^{144}$

Als Ergebnis kann daher festgehalten werden, daß die in der Charta in den Art. 2, 19 festgelegten institutionellen Regelungen zur Streitbeilegung mangels rechtlicher Verbindlichkeit ineffektiv und in der Praxis leergelaufen sind. Die fehlende institutionelle, rechtlich verbindliche Möglichkeit zur Streitbeilegung bedauert auch Cervenka, wenn er schreibt: "The logical consequence of absence of any central authority within the OAU, which would be vested with executive powers, was the absence of any kind of effective extinguisher of hostilities of which the machinery for the peaceful settlement of disputes

$141 \mathrm{Vgl}$. Elias, The commission of mediation, conciliation and arbitration of the Organization of African Unity, in: British Yearbook of International Law, (1964), S. 336-354 und Jonah, The U.N. and the O.A.U.: Roles in the maintenance of international peace and security in Africa, in: El-Ayouty, Brooks (Hg.), a.a.O. (Anm. 94), S. 127-151 (141ff.).

142 Vgl. Cervenka, in: El-Ayouty, Brooks (Hg.), a.a.O. (Anm. 94), S. 59ff.

143 Ebenso Elias, a.a.O. (Anm. 141), S. 343 und Kunig, a.a.O. (Anm. 2), S. $46 f$.

144 Vgl. Jonah, a.a.O. (Anm. 141), S. 127-151(143). 
would be an indivisible part. "145 Für Cervenka war es daher keine Uberraschung, daß der erste zwischen OAU-Mitgliedern entstandene Streit nach der Gründung der OAU die Organisation völlig unvorbereitet fand, um sich mit ihm zu befassen. Wenngleich eine erste erfolgreiche Friedensinitiative im Grenzkrieg zwischen Marokko und Algerien 1963 von Äthiopien und Mali ausging, so wurde das positive Ergebnis doch als Verdienst der OAU gefeiert, dessen Ministerrat dann auch Marokko und Algerien "für ihr Vertrauen in die OAU" dankte.

Um nach der ersten, in Form eines Kommuniqués bekräftigten Ubereinkunft zwischen Marokko und Algerien eine dauerhafte Lösung im Grenzkonflikt herbeizuführen, regten die Streitparteien selbst erfolgreich die Gründung einer in der Charta nicht vorgesehenen ad-hoc-Kommission an. Aus sieben Mitgliedern bestehend trat eine solche noch im Dezember 1963 zusammen, entschied über die Richtlinien und Form seiner Arbeit selbst und wirkte im Sinne der vom Ministerrat übertragenen Aufgabe als "Committee of arbitration for the definite settlement of the Algerian-Moroccan dispute". Wenngleich die Aufgaben und Befugnisse dieser ad-hoc-Kommission nicht festgelegt waren und sich auch die Kommissionsmitglieder selbst im unklaren darüber waren, so hat sich in diesem Fall die Arbeit der Kommission abschließend doch als erfolgreich erwiesen: Der Konflikt wurde beigelegt. ${ }^{146}$ Noch bevor sich die in Art. 19 der Charta vorgesehene Kommission konstituieren konnte, hatte sich eine bei Bedarf zusammentretende und den Gegebenheiten entsprechend zusammensetzbare ad-hoc-Kommission bereits etabliert, ohne eine Rechtsgrundlage in der Satzung zu haben. ${ }^{147}$ Auch nach 1963 folgende zahlreiche bilaterale Konflikte wurden von der OAU behandelt, oder sich auf die OAU-Mitgliedschaft beziehende Staaten nahmen sich ihrer auf diplomatischen Wegen vermittelnd an und leisteten gute Dienste, ohne daß diese Vorgehensweise formell und die inhaltlichen Vermittlungsvorschläge materiell behandelt und geregelt waren. Wie nützlich die Ausarbeitung von Regeln, wenn nicht im Sinn verbindlicher Rechtssätze, so doch als empfehlende Richtlinien oder Grundsätzen wären, zeigt das Beispiel der in der Folge des algerisch-marokkanischen Disputes verabschiedeten, die Präambel und die Artikel der Charta auslegenden Resolution über die Respektierung der Kolonialgrenzen. In der Erkenntnis, daß die Grenzfrage ein "ständiger bedeutsamer Gegenstand von Auseinandersetzungen" sein würde, beinhaltet sie, "that all Member States pledge themselves to respect the borders existing on their achievement of national independence" ${ }^{148}$ Diese Resolution hat faktisch durch allgemeine Anerkennung die Qualität eines Rechtssatzes bekommen, der grundsätzlich von den Mitgliedstaaten respektiert wird. In der Vergangenheit hat diese klare materielle Festlegung zur Hilfestellung im Sinn

145 In: El-Ayouty, Brooks (Hg.), a.a.O. (Anm. 94), S. 53.

$146 \mathrm{Vgl}$. Berko Wild, The Organization of African Unity and the algerian-moroccan border conflict, in: International Organization, XX(1966)I, S. 18-36.

147 Vgl. Kunig, a.a.O. (Anm. 25), S. 135,140f.

148 Assembly of Heads of State and Government/Resolution 16/I, 1964 (Kairo). 
eindeutiger Haltung der OAU und daraus folgender aktiver Haltung bei Grenzkonflikten geführt.

In anderen Fällen, in denen Krisen nicht durch zuvor entwickelte eindeutige formelle und materielle Regelungen durch die OAU verhindert oder beendet werden konnten, hat sich die Unfähigkeit der OAU zur Konfliktbeilegung manifestiert. So war die OAU diskreditiert, als ihr Ministerrat 1965 im Zusammenhang mit der Rhodesienfrage mehrheitlich den Abbruch diplomatischer Beziehungen der Mitglieder zu Großbritannien empfahl, aber sich 2/3 der Mitglieder nicht an den Beschluß hielten, den ihre eigenen Minister verabschiedet hatten. Ein weiterer Beleg für diese Einschätzung ist die Untätigkeit der OAU, nachdem während allgemeiner Boykottaufrufe gegenüber der Republik Südafrika das OAU-Mitglied Malawi Südafrika 1967 anerkannte; ein Ausschlußantrag Sambias blieb unbeachtet. Ein Ausschluß eines Mitglieds ist allerdings seit der Gründung der OAU noch in keinem Fall vollzogen worden. Es wird unabhängig von der Frage nach einer Rechtsgrundlage in der Satzung befürchtet, daß der Ausschluß eines Mitglieds wegen fehlender Prinzipientreue in einem Punkt in der Zukunft leichter zu weiteren Ausschlüssen wegen mangelnder Prinzipientreue in anderen Punkten führen kann. Eine restriktive Auslegung der Mitgliedschaftsbedingungen der OAU kann so leicht zu einer Hexenjagd auf Mitglieder führen, mit verheerenden Folgen für die Organisation. ${ }^{149}$

Eines der augenfälligsten Beispiele für das Unvermögen der OAU ist ihre Rolle anläßlich der 2. Kongo-Krise von 1964, als die Interessengegensätze zwischen der ehemaligen Casablanca- und der Brazzaville-Gruppe wieder aufbrachen und trotz größten Engagements durch das Sekretariat, den Ministerrat und einem ad-hoc-Komitee die Gegensätze nicht überbrückt und die Krise durch die OAU nicht abgewendet werden konnte. Wallerstein sieht den Grund des Scheiterns hier nicht im Mangel an rechtlicher Kompetenz, sondern letztlich im Fehlen von tatsächlicher Macht. So beschließt Wallerstein seine Untersuchung mit der Erkenntnis: "What the OAU has not been able to do is impose its will or even its effective presence when the interest of a Member State went strongly in another direction ". ${ }^{150}$

Mit Ortiz de la Torre ist abschließend feststellbar, daß "die Kapazitäten der OAU . . . noch mehr als die der UNO eingeschränkt sind, um die im internationalen Recht niedergelegten Rechte zu konsolidieren ". ${ }^{151}$

Die bisherige Geschichte der Sahara hat gezeigt, daß es die OAU nicht vermochte, den Sahraouis zur Durchsetzung ihres Selbstbestimmungsrechts zu verhelfen und auf diesem Wege zu einer friedlichen Streitbeilegung beizutragen. Es bleibt abzuwarten, ob eine in letzter Zeit verschiedentlich geforderte Novelle der OAU-Satzung den beschriebenen Mängeln wird abhelfen können.

149 So Tandon, Die Organisation der Afrikanischen Einheit in einer Phase der Konsolidierung, in: Europa-Archiv, 23(1971), S. 835-842(842).

150 A.a.O. (Anm. 94), S. 27.

151 A.a.O. (Anm. 19). 


\title{
The Silent Reality: Racial Discrimination in the Criminal Justice Process
}

\author{
By Lovell D. Fernandez
}

The study shows race to be an important variable influencing the outcome of South African criminal trials. The racial discriminatory laws and the way they are implemented ensure that the black population in particular remains in constant contact with the criminal justice system. The court-record study shows that black persons charged with capital offences are denied a chance to defend themselves effectively: either because they are subject to the whims of low-ranking administration officials. The latter is especially manifest in the realm of state-endangering crimes where the Executive enjoys considerable latitude in its dealings with detainees and accused persons. An obligation rests on the legal profession to address itself earnestly to the inequities in the criminal justice system. The plea is for a humane justice. Lawyers should resist attempts to bring the judiciary under the control if the Executive. Furthermore, an urgent need exists to expand the ambit of criminal legal aid.

\section{The Sahara Conflict and the Crisis of the Organization of African Unity}

\section{By Jürgen Taeger}

The Organization of African Unity (OAU) which has the most individual members of any regional international organization is now experiencing the greatest test of its strength and effectiveness in its 20 -year history.

In 1975, through the tripartite Treaty of Madrid, Spain transferred possession of the colony to Morocco and Mauritania. This action was in itself a breach of the existing Decolonization Rules. Since then the population of the Western Sahara has been struggling to achieve self-determination. In 1976, following the withdrawal of the colonial power Spain, a new state, the Saharan Arab Democratic Rebuplic (SADR), was founded.

After the signing of a peace treaty between the SADR and Mauritania, Morocco demanded possession of the entire Sahara and proceeded to occupy the so-called wuseful triangle", an area in the northwest of the Sahara which is very rich in raw materials. The present economic situation in Morocco is catastrophic; the Moroccan king, Hassan II, is able to continue his war against the Saharan Independence Movement, the POLISARIO Front, only with massive economic and military support from the United States.

For years, Hassan II has successfully employed delaying tactics to frustrate the attempts of international organizations, especially of the OAU, to find a diplomatic solution to the conflict. Despite these tactics, Hassan II was unable to prevent admission of the SADR as the 52 nd member of the OAU. The monarch and sympathetic African heads of state are now threatening to boycott the OAU. Hassan's efforts did prevent a referen- 
dum regarding the future of the Sahara even though the OAU was strongly in favour of holding one, in 1983.

In the face of the rejection of the referendum, the leaders of African states who plan to gather for a summit meeting in Conakry, Guinea, in the summer of 1984 face extremely difficult decisions, especially because the representatives of the SADR now demand to take their seats in the OAU after years of willingness to compromise.

This article sketches the historical development and the present position on self-determination in the SADR. The foreign relations of the SADR are specifically addressed and efforts to peacefully settle the conflict are critically examined. 